\title{
Molecular characterization of polyOAPS-imide isomer hyper-cross-linked membranes: Free-volume morphologies and sorption isotherms for $\mathrm{CH}_{4}$ and $\mathrm{CO}_{2}$
}

\author{
Sylvie Neyertz ${ }^{\mathrm{a}, *}$, David Brown ${ }^{\mathrm{a}}$, Saman Salimi ${ }^{\mathrm{a}}$, Farzaneh Radmanesh ${ }^{\mathrm{b}}$, Nieck E. Benes ${ }^{\mathrm{b}}$ \\ ${ }^{a}$ Univ. Savoie Mont Blanc, Univ. Grenoble Alpes, CNRS, Grenoble INP, LEPMI, 38000, Grenoble, France \\ ${ }^{\mathrm{b}}$ Films in Fluids, Department of Science and Technology, MESA+ Institute for Nanotechnology, University of Twente, P.O. Box 217, 7500, AE Enschede, the Netherlands
}

\section{A R T I C L E I N F O}

\section{Keywords:}

Hyper-cross-linked hybrid networks PolyOAPS/POSS-Imides

Molecular dynamics (MD) simulations

Grand canonical Monte Carlo(GCMC)-MD simulations

Free-volume morphology

$\mathrm{CH}_{4}$ and $\mathrm{CO}_{2}$ sorption

\begin{abstract}
A B S T R A C T
PolyPOSS-imides are hybrid hyper-cross-linked networks based on inorganic octa(aminopropyl)silsesquioxane (POSS) cages covalently bonded via organic imide bridges. They were initially developed for separations under harsh conditions, but they cannot be used above $300{ }^{\circ} \mathrm{C}$ due to the decomposition of their $-\left(\mathrm{CH}_{2}\right)_{3}-$ propyl linkers. Replacing the aliphatic-arm POSS with its phenyl-arm derivative, octa(aminophenyl)silsesquioxane (OAPS), should thus increase their resistance. However, OAPS has three possible isomers depending on the meta, para or ortho position of the $-\mathrm{NH}_{2}$ group on the phenyl ring. To pre-screen the most interesting structures before attempting synthesis, molecular dynamics (MD) simulations were recently used to compare twenty-two model networks based on the OAPS isomers, the initial POSS and several imide precursors at high temperatures. Six phenyl-arm polyOAPS-PMDA and polyOAPS-6FDA and two aliphatic-arm polyPOSS-PMDA and polyPOSS-6FDA were selected for further analyses of their $\mathrm{CH}_{4}$ and $\mathrm{CO}_{2}$ sorption isotherms at $35^{\circ} \mathrm{C}$. All of them have an average number of $\sim 5$ links per cage and cross-linking densities of $\sim 3.8$. In terms of connectivities and free-volume morphologies, both the metaOAPS and paraOAPS networks are found to be open structures with many lowenergy sites and high penetrant solubilities. On the other hand, the ortho functionalization leads to denser morphologies associated to lower solubilities, which makes it closer to the POSS networks. Differences are enhanced when the inorganic precursor is associated to a rigid organic linker such as PMDA.

The iterative GCMC (Grand Canonical Monte Carlo)-MD sorption-relaxation technique was used to predict the single-gas uptakes of $\mathrm{CH}_{4}$ and $\mathrm{CO}_{2}$ in the networks over an extended $0-60$ bar pressure range at $35{ }^{\circ} \mathrm{C}$. The sorption capacities varied in the order paraOAPS $>$ metaOAPS $>$ orthoOAPS $>$ POSS for the inorganic precursor, and PMDA $>6$ FDA for the organic precursor (except for the orthoOAPS). All correlate directly to the accessible free-volume, but the $\mathrm{CO}_{2} / \mathrm{CH}_{4}$ ideal sorption selectivities remained essentially the same over the whole pressure range. Compared to POSS, OAPS showed less swelling at equivalent penetrant concentrations in the matrix. It is also more resistant to other mechanical deformations such as isotropic dilation or uniaxial tension. This confirms that the polyOAPS-imides can indeed be used under harsher conditions than the current polyPOSS-imides, without loss of the $\mathrm{CO}_{2} / \mathrm{CH}_{4}$ sorption selectivities.
\end{abstract}

\section{Introduction}

The separations of mixtures account for over $50 \%$ of the energy costs in chemical industries [1]. They are particularly difficult to carry out when they involve molecules of a similar size such as small gases and vapours. Dense polymer membranes have long been considered as a viable alternative to the traditional cryogenic distillation or selective adsorption processes because of their ability to separate small penetrants based on their differences in solubilities and/or diffusivities $[2,3]$. Their energy cost is estimated to be $90 \%$ less than distillation and the membrane devices are easy to scale-up. However, in practice, their use is still under-developed because of the need to find more resistant materials $[1,4]$. While many polymers have been considered for their transport properties, these are usually assessed under moderate temperatures and pressures [5]. On the other hand, when exposed to harsher conditions, conventional polymers tend to lose their structural integrity and, as a result, their permselectivities. This is problematic since many

\footnotetext{
* Corresponding author. LEPMI, CNRS UMR 5279, Univ. Savoie Mont Blanc, Bât. Hélios, Savoie Technolac, 73376, Le Bourget du Lac Cedex, France.

E-mail address: Sylvie.Neyertz@univ-smb.fr (S. Neyertz).
} 


\begin{tabular}{|c|c|c|c|}
\hline \multicolumn{2}{|c|}{ Nomenclature } & $V$ & volume \\
\hline$C_{\text {gas }}$ & concentration of the penetrant in the gas phase & & method \\
\hline$C_{\text {matrix }}$ & concentration of the penetrant in the matrix phase & $\alpha^{\mathrm{S}}$ & ideal sorption selectivity \\
\hline FSV & fraction of significant volume & $\mu_{\text {gas }}$ & chemical potential of the penetrant molecule in the gas \\
\hline$k_{B}$ & Boltzmann constant & & phase \\
\hline $\begin{array}{l}\boldsymbol{P} \\
p\end{array}$ & $\begin{array}{l}\text { pressure tensor } \\
\text { pressure }\end{array}$ & $\mu_{\text {matrix }}$ & $\begin{array}{l}\text { chemical potential of the penetrant molecule in the matrix } \\
\text { phase }\end{array}$ \\
\hline$P A V$ & probe-accessible volume & $\Delta \Phi$ & change in potential energy for the (virtual) insertion of a \\
\hline$p_{i g}$ & probability of an insertion in the gas phase & & test particle \\
\hline $\begin{array}{l}p_{\text {im }} \\
S_{\text {gas }}\end{array}$ & $\begin{array}{l}\text { probability of an insertion in the matrix phase } \\
\text { solubility of the penetrant in the gas phase }\end{array}$ & $\Delta \Phi_{\mathrm{c}}$ & $\begin{array}{l}\text { critical upper limit in the insertion energy used to define } \\
\text { the FSV }\end{array}$ \\
\hline$S_{\text {matrix }}$ & solubility of the penetrant in the matrix phase & $\rho(\Delta \Phi)$ & normalized probability density of $\Delta \Phi$ \\
\hline$T$ & temperature & $\rho_{w}(\Delta \Phi)$ & Boltzmann-weighted normalized probability density of $\Delta \Phi$ \\
\hline$u_{b}$ & unbiaising factor in the EVMS method & & \\
\hline
\end{tabular}

industrial treatments of natural gas, syngas or power plant flue gas involve high-pressure feeds and/or high temperatures [6,7].

Hybrid organic-inorganic materials have emerged as promising candidates for improving the thermomechanical resistance of separation membranes while maintaining their gas-sieving properties [8]. Hybrids based on the cubic inorganic polyhedral oligomeric silsesquioxanes (POSS), i.e. $\mathrm{Si}_{8} \mathrm{O}_{12}$ siloxane cages with 8 organic arms $R$ attached to the silicium atoms [9-11], are particularly interesting because of their high versatility. Depending on the nature of $R$, they can either be physically mixed or chemically cross-linked to a large number of polymers [12-16]. A recent review reports the many opportunities for POSS in the field of advanced separation membranes [17].

The focus here is on hyper-cross-linked polyPOSS-imide networks [18-21], in which amino-functionalized inorganic POSS are covalently bonded with organic imides originating from dianhydride precursors. It should be noted that dianhydrides can also act as precursors for organic polyimides, which usually show good gas selectivities [22,23]. In the case of the hybrid networks, solutions of both types of precursors are prepared at room temperature in non-miscible solvents. When contacted, interfacial polycondensation occurs between the $-\mathrm{NH}_{2}$ on the POSS and the anhydride moieties. Since both sides of the dianhydrides are able to react, this results in a defect-free hyper-cross-linked polyPOSS-(amic acid) film, which is further converted into its final polyPOSS-imide form via thermal imidization at $300{ }^{\circ} \mathrm{C}$. This procedure can be directly carried out on high-surface-to-volume ratio hollow fibre supports for upscaling [24], and as such, it is well adapted to membrane modules [25].

The first polyPOSS-imides developed by interfacial polycondensation were based on octa(aminopropyl)silsesquioxane, i.e. a POSS precursor functionalized with aliphatic $-\left(\mathrm{CH}_{2}\right)_{3}-\mathrm{NH}_{2}$ arms (Fig. 1a) [18-21]. They were indeed found to perform under tougher operating conditions (up to 60 bar and $300{ }^{\circ} \mathrm{C}$ ) than conventional polymer membranes [18-20,25]. However, the flexible aliphatic arms of the POSS resulted in excessive dilations and thermal degradation above $\sim 300{ }^{\circ} \mathrm{C}$, thus limiting the gas separation efficiencies of the imide constituents. This was confirmed by molecular simulations [26-28]. It was then proposed to replace the initial POSS precursor by octa(aminophenyl)silsesquioxane, commonly referred to as OAPS [10,29], which is functionalized by phenyl $-\mathrm{C}_{6} \mathrm{H}_{4}-\mathrm{NH}_{2}$ arms [30]. There are three isomers, since the $-\mathrm{NH}_{2}$ group can be attached at different positions on the phenyl ring, and we refer to them as metaOAPS, paraOAPS and orthoOAPS. The three OAPS isomers can co-exist in various proportions depending on the synthesis route $[29,31,32]$, but they can also be obtained separately from the appropriate aminophenyltrialkoxysilanes [33]. Our model networks were based on each of the different isomers to assess unambiguously the effects of the substitution position. Their chemical structures are given in Fig. 1a along with those of the initial

\section{POSS precursor.}

Fig. 1b shows the organic precursors under study, i.e. the pyromellitic dianhydride (PMDA) and the 4,4'-(hexafluoroisopropylidene) diphthalic dianhydride (6FDA). Hyper-cross-linked networks based on the OAPS inorganic precursor are referred to as "polyOAPS-imides" to differentiate them from the "polyPOSS-imides" based on the initial aliphatic POSS precursor, and two of their chemical structures are provided in Fig. 1c-d.

When attempting to develop new materials, molecular modelling techniques such as molecular dynamics (MD) and Monte Carlo (MC) simulations, can be used to pre-screen a large series of candidate structures [34]. This allows for the subsequent synthesis efforts to only concentrate on the most promising ones. Furthermore, experiments under harsh conditions are difficult to implement and to interpret [35], whereas both MD and MC simulations can be carried out under a large variety of operating pressures and temperatures [36]. The present work represents the third stage in the detailed molecular characterization of polyPOSS/OAPS-imides. In a first stage [26-28], the generation procedure for such hyper-cross-linked model networks was optimized on several polyPOSS-PMDA and polyPOSS-6FDA at the experimentally-determined initial average value of 4 links per POSS cage [19]. This allowed for the first detailed characterization of their structures, thermomechanical properties, and $\mathrm{CO}_{2}$ and $\mathrm{CH}_{4}$ permeabilities at the molecular level [26-28]. Following the identification of the POSS aliphatic arms as a weak link, eighteen new molecular models were generated based on the three isomers of OAPS as the inorganic precursors and on the PMDA, 6FDA and 4,4'-oxydiphthalic (ODPA) dianhydrides as the organic precursors [30]. The cross-linking procedure was stopped at the same average value of 4 links per OAPS cage for nine of them, but the remaining nine polyOAPS-imides were allowed to cross-link until there were no more possible reactions. Their characteristics and thermomechanical resistances were then directly compared to those of the corresponding polyPOSS-imides at $300{ }^{\circ} \mathrm{C}$, i.e. at the experimental temperature for the imidization. While the onset of decomposition for polyPOSS-imides is known to be at $\sim 350^{\circ} \mathrm{C}$ [21], that of polyOAPS-imides is expected to be well over $400^{\circ} \mathrm{C}[37,38]$. Carrying out the cross-linking reactions to completion significantly improved the resistance. Indeed, following the initial characterization of 4 links per cage on average [19], it was later shown experimentally that increasing the concentration of the POSS solution could lead to connectivities of up to 4.9 links per cage for polyPOSS-6FDA [20]. This is very similar to the value found when carrying out the cross-linking to completion for our model polyPOSS-6FDA. Furthermore, the polyOAPS-imides with the maximum degree of connectivity were found to maintain their structural integrities at $400{ }^{\circ} \mathrm{C}$. In all cases, the optimal networks were those either based on the orthoOAPS inorganic precursor and/or on the rigid PMDA organic precursor with the maximum possible connectivity. Those based 
(a) inorganic precursors

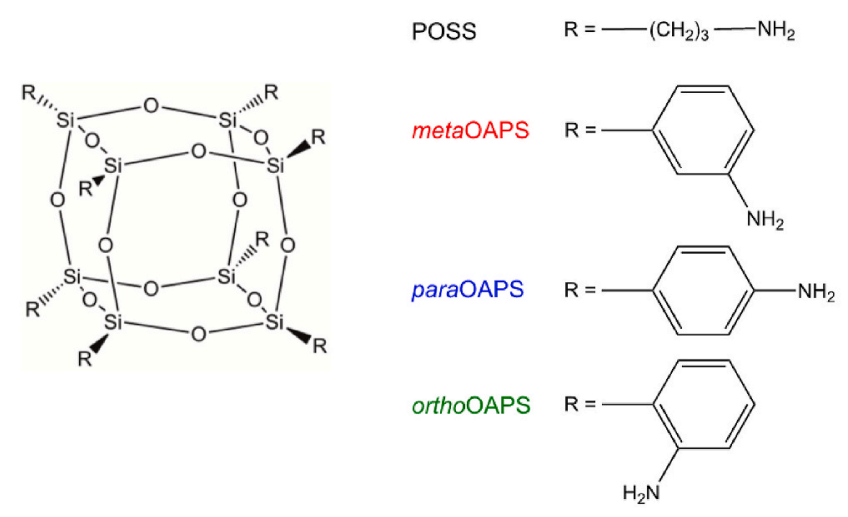

(b) organic precursors<smiles>O=c1oc(=O)c2cc3c(=O)oc(=O)c3cc12</smiles>

PMDA<smiles>O=C1OC(=O)c2cc(C(c3ccc4c(c3)C(=O)OC4=O)(C(F)(F)F)C(F)(F)F)ccc21</smiles>

6FDA

(c) poly orthoOAPS-PMDA

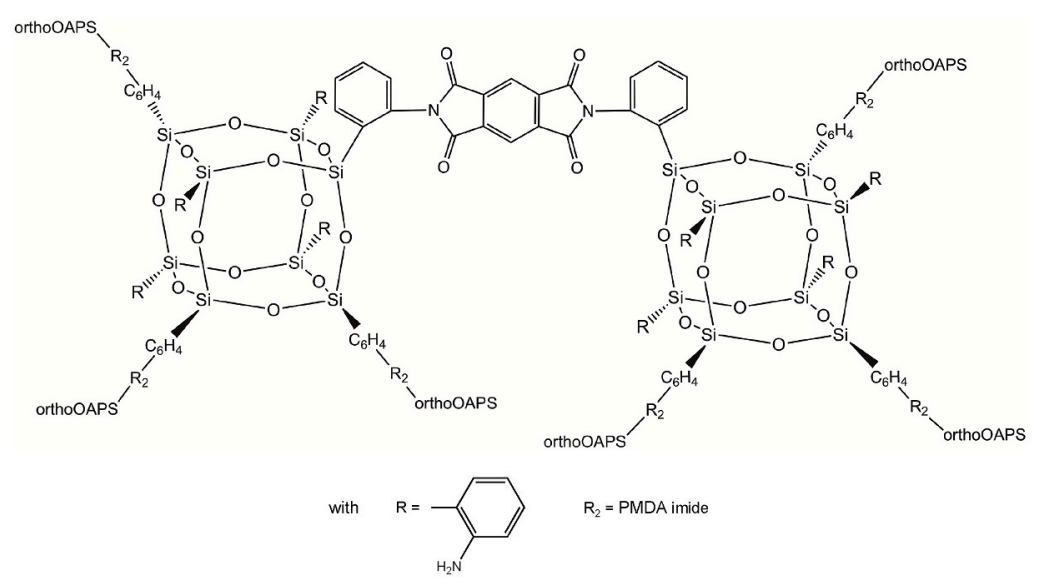

(d) polyparaOAPS-6FDA

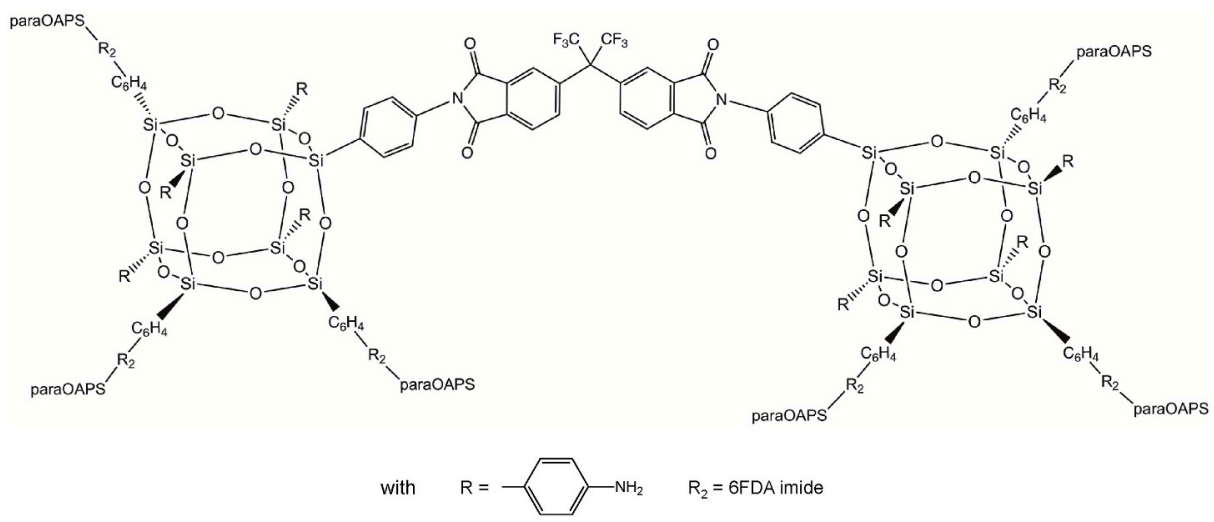

Fig. 1. (a) Inorganic and (b) organic precursors for the hyper-cross-linked polyPOSS-imides and polyOAPS-imides under study. (c) and (d) show two polyOAPSimide networks with an average connectivity of four links per OAPS cage. 
on the metaOAPS and paraOAPS (with the same organic precursor) behaved in a fairly close way, as did those based on the two flexible organic linkers, 6FDA and ODPA (with the same inorganic precursor) [30].

The present work investigates the behaviour and the ideal $\mathrm{CO}_{2} / \mathrm{CH}_{4}$ sorption selectivities at $35{ }^{\circ} \mathrm{C}$, i.e. just above the critical temperature for $\mathrm{CO}_{2}$, for the most promising polyOAPS-imide networks selected over the aforementioned screening stage [30]. Since bulk periodic molecular models do not have any explicit external reservoirs, specific techniques are required to estimate the number of penetrant molecules in the networks in equilibrium with a preset external gas pressure [36]. The most commonly-used approach is the efficient Grand Canonical Monte Carlo (GCMC) method [34,39-44], but the calculations have to be carried out on a set of pre-prepared matrix configurations. It can result in artefacts upon loading of either the mildly-plasticizing $\mathrm{CH}_{4}$ or, even worse, the highly-plasticizing $\mathrm{CO}_{2}$ which leads to significant matrix dilations [45]. This problem can be addressed by coupling GCMC with controlled-pressure MD, which naturally allows for the matrix and its volume to relax upon penetrant sorption $[34,42,45-50]$. However, the decreasing density during the MD phase results in the penetrant solubility rising. The model thus drifts from the preset external gas pressure and it is necessary to reapply GCMC to adjust the sorbed number of molecules corresponding to the newly-relaxed volume. Several alternating cycles of sorption and relaxation are usually required to reach convergence and, as such, we refer to this sorption-relaxation procedure as the "iterative GCMC-MD method" [36]. Due to the slow molecular relaxations [28], a recent work has shown that 30-40 GCMC cycles coupled with controlled-pressure MD simulations of 1000 ps were required to attain convergence for $\mathrm{CO}_{2}$ in the $6 \mathrm{FDA}-6 \mathrm{FpDA}$ polyimide [36]. It is clear that these are very time-consuming simulations and consequently, the number of networks under study was limited to eight. GCMC on its own is more appropriate for rapid screening of the sorption capacities $[34,51]$, but it has to be restricted either to low-plasticizing penetrants or to low uptakes for plasticizing penetrants. In the present case, we assess $\mathrm{CH}_{4}$ and $\mathrm{CO}_{2}$ sorption over an extended 0-60 bar range at $35{ }^{\circ} \mathrm{C}$, and as such, we need to make extensive use of the iterative GCMC-MD method.

The selected networks were the three polyOAPS-PMDA and the three polyOAPS-6FDA, each of them with a different OAPS isomer, with the cross-linking reactions carried out to completion, along with a polyPOSS-PMDA and a polyPOSS-6FDA generated under exactly the same conditions [30]. The inclusion of these latter samples was the reason for the choice of the polyOAPS-6FDA over the polyOAPS-ODPA (which behaved quite similarly), the main points being that i) the OAPS-based networks can be compared to the corresponding POSS-based and ii) the networks with the rigid PMDA organic linker can be compared to those with a flexible linker. The details of the models and the specific methodologies associated to gas solubility and sorption are summarized in Section 2 and Section 3, respectively. Section 4 analyses the bulk properties and the free-volume morphologies of the pure networks at $35^{\circ} \mathrm{C}$. Section 5 compares their uptake curves for both $\mathrm{CH}_{4}$ and $\mathrm{CO}_{2}$, their amount of swelling, their interactions with the gases as well as their ideal $\mathrm{CO}_{2} / \mathrm{CH}_{4}$ selectivities. Their resistance to isotropic dilation and uniaxial tension is discussed in Section 6.

\section{Network molecular models}

As mentioned above, the polyPOSS-imide and polyOAPS-imide model networks have been presented in detail before [26-28,30], but for clarity, we hereby summarize their main features.

\subsection{Force-fields and simulation parameters}

Both MD and GCMC simulations were carried out using the gmq parallel package [52]. The total molecular potential energy $U_{p o t}$ is obtained from the sum of the angle-bending, torsional, out-of-plane, van der Waals and electrostatic interactions (Eq. (1)):

$U_{\text {pot }}=\sum_{\theta} U_{\text {bend }}(\theta)+\sum_{\tau} U_{\text {tors }}(\tau)+\sum_{i-\text { planar }} U_{\text {oop }}(i)+\sum_{(i, j) n b} U_{\text {vdw }}(r)+\sum_{(i, j) n b} U_{\text {coul }}(r)$

Each term in Eq. (1) is detailed in the Supporting Information, along with the force-field parameters for all OAPS-based molecules (Tables S1S2-S3 and Fig. S1) and the cutoffs for non-bonded interactions [30]. Those for the aliphatic POSS-based molecules are available in Refs. [26, 27]. The gas molecules were represented by optimized all-atom models for $\mathrm{CH}_{4}$ [53] and $\mathrm{CO}_{2}$ [54]. High-frequency motions, i.e. the bond stretching modes, the hydrogen vibrational modes and the $\mathrm{O}=\mathrm{C}=\mathrm{O}$ bending mode for $\mathrm{CO}_{2}$, were kept rigid using constraints [55,56], in order to ensure a correct equipartition of the kinetic energy with a time step of $\Delta t=10^{-15} \mathrm{~s}$ in the MD integration algorithm [57].

The temperature $T$ and pressure tensor $\boldsymbol{P}$ were maintained close to their required values by loose-coupling $[58,59]$. The MD simulations were run under either constant-volume NVT or controlled-pressure-tensor NPT conditions. The latter allows for the system to relax towards its equilibrium size and shape. The resistances to mechanical stresses were obtained by changing linearly the components of the required pressure tensors and recording the changes in box sizes and shapes. The average thermodynamic data were stored every $1 \mathrm{ps}$ while the molecular configurations were stored every 5 ps for post-analysis. They were visualized with the VMD software [60].

\subsection{Generation and relaxation of the hyper-cross-linked networks}

The fully-atomistic networks were built using a similar procedure to other molecular models of cross-linked materials [61-72]. It basically starts with mixtures of the precursors, in which cross-links are formed using a heuristic distance criteria and are subsequently relaxed [70]. The composition of the mixtures, the distance criterion, the target cross-linked densities and the relaxation protocol are usually chosen to mimick experiment. The synthesis route for the hyper-cross-linked networks under study here [18-21] is provided in Fig. S2 of the Supporting Information.

A detailed description of the optimized generation procedure for our polyOAPS-imides and polyPOSS-imides is available in Ref. [30]. To summarize, separate bulk models of the different inorganic and organic precursors (Fig. 1) were first built and validated at room temperature. 3:1 dianhydride:OAPS and dianhydride:POSS mixtures were then prepared by superimposing an inorganic bulk with an organic bulk and selecting the molecules that overlapped the least. Using 216 OAPS (or POSS) precursors led to model networks of $\sim 30000-40000$ atoms, i.e. they were large enough to give statistically-significant results $[66,68]$. The mixtures were relaxed at $300{ }^{\circ} \mathrm{C}$, cooled back to room temperature, and carefully checked for any signs of phase separations. This did not occur and the cross-linking started with recording all the distances between the dianhydride ketone carbons $\mathrm{C}_{\mathrm{ket}}$ and the OAPS (or POSS) amine nitrogens $\mathrm{N}$. Based on these $\mathrm{C}_{\mathrm{ket}} \cdots \mathrm{N}$ radial distribution functions (Fig. S3 in the Supp. Information), the maximum distance criteria for the sum of the shortest $\mathrm{C}_{\mathrm{ket}} \cdots \mathrm{N}$ distances at either end of a dianhydride, $R_{\text {min }}$, was set to $7 \AA$. Earlier investigations have shown that setting $R_{\text {min }}$ to either 6 or to $7 \AA$ gave similar connectivities for the polyPOSS-imides $[26,27]$, but that $6 \AA$ is too short for dianhydrides to be able to cross-link with the orthoOAPS precursor [30]. As such, all dianhydrides having $R_{\min } \leq 7 \AA$ were selected, both their covalent bonds between $\mathrm{C}_{\mathrm{ket}}$ and the anhydride oxygen $\mathrm{O}_{\text {anhy }}$ were broken and replaced by two new $\mathrm{C}_{\text {ket }} \mathrm{N}$ bonds. $\mathrm{O}_{\text {anhy }}$ and the amine hydrogens were removed, thus mimicking the experimental loss of one water molecule per imidization reaction [18-21]. The newly created OAPS-diimide (or POSS-diimide) links were relaxed back to their equilibrium bond lengths by energy minimization, and the network was gradually re-thermalized with MD under NVT conditions up to $10 \mathrm{ps}$. At that point, $R_{\min }$ was re-calculated for all unreacted dianhydrides, and those within the $R_{\min } \leq 7 \AA$ criterion were 
selected to form more bonds. The cross-linking-relaxation process continued in an iterative manner until it was terminated and the remaining unreacted dianhydrides were then removed from the system. The cross-linking reactions can either be stopped once a specific degree of connectivity is reached or continued until there are no more possible reactions.

Our screening of 22 different structures [30] showed that the networks with the reactions carried out to completion exhibit significantly higher resistances to mechanical deformation than those with slightly lower cross-linking densities. The former required much longer cross-linking simulation times, as it became less and less frequent to find unreacted dianhydrides with $R_{\min } \leq 7 \AA$. The increased degree of cross-linking, however, resulted in over $99 \%$ of the atoms forming a single continuous network with the few remaining molecules belonging either to unreacted cages or to small cage-dianhydride-cage blocks. All the networks considered here will thus be those obtained by carrying out the reactions to completion, i.e. those with the higher degrees of connectivity.

Once cross-linked, the networks were relaxed for 20000 ps under $N P T$ conditions at $300{ }^{\circ} \mathrm{C}$ to mimick the experimental imidization conditions. The initial screening stage [30] was directly carried out at $300{ }^{\circ} \mathrm{C}$, since these networks are aimed at being resistant under harsh conditions. The structures were then cooled down at $-0.1 \mathrm{~K} / \mathrm{ps}$ and further run at $35{ }^{\circ} \mathrm{C}$ for $4000 \mathrm{ps}$. The densities and energies relaxed fast (see Fig. S4 in the Supp. Info) and the properties at $35{ }^{\circ} \mathrm{C}$ could be averaged over the last $2000 \mathrm{ps}$. It should be noted that there is always a dependence of the density on the cooling rate [73], but this usually remains minor with $-0.1 \mathrm{~K} / \mathrm{ps}$, which is a fairly slow cooling rate for MD simulations [36].

\section{Gas solubility and sorption methodologies}

\subsection{The TPI method for gas solubility}

In molecular simulations, the free energy for the solvation process is usually estimated using the test-particle insertion (TPI) approach [74, 75]. This means repeatedly inserting a virtual penetrant molecule at random sites into a matrix, here the hyper-cross-linked networks, and calculating the average change in the potential energy $\Delta \Phi$ associated with million of trials insertion. The (dimensionless) solubility $S$ can be obtained in the NPT ensemble from the following equation [76]:

$S=\frac{\left\langle V \exp \left(-\frac{\Delta \Phi}{k_{B} T}\right)\right\rangle}{\langle V\rangle} \approx\left\langle\exp \left(-\frac{\Delta \Phi}{k_{B} T}\right)\right\rangle$

with $k_{\mathrm{B}}$ being the Boltzmann constant. In the case of limited volume fluctuations, the approximation given in Eq. (2) holds quite well. To improve its efficiency [36], the TPI method can be parallelized and combined with an excluded-volume map sampling (EVMS) approach, which screens out regions of very low insertion probabilities [77-79]. For dense matrices, this allows for up to $85-95 \%$ of the space to be pre-excluded, thus speeding-up TPI by factors of 10-20 compared to purely random insertions $[28,80,81]$.

The probability density distributions of the insertion energies $\rho(\Delta \Phi)$ can be weighted by their Boltzmann factor to represent the likely energies for any probe molecule actually inserted into the matrix, i.e. the preferential sites for penetrant adsorption [28]:

$\rho_{w}(\Delta \Phi)=\rho(\Delta \Phi) \exp \left(-\frac{\Delta \Phi}{k_{B} T}\right)$

The $\rho_{w}(\Delta \Phi)$ can also be used to check the reliability of TPI as poorlyconverged unweighted $\rho(\Delta \Phi)$ give rise to ragged Boltzmann-factor weighted distributions [28].

In the present work, the parallel TPI-EVMS was used to characterize the solubility of $\mathrm{CH}_{4}$ and $\mathrm{CO}_{2}$ in the pure networks and the morphology of their respective void-spaces. It was also used prior to the uptake curve predictions, as the latter require for each gas the determination of its concentration $C_{g a s}$ and its solubility $S_{g a s}$ in its own gas phase over the full range of pressures $p$ considered. For the models of $\mathrm{CH}_{4}$ and $\mathrm{CO}_{2}[53,54]$, the $C_{g a s}(p)$ and the $S_{g a s}(p)$ were previously obtained from a series of MD simulations of the pure gas under $N V T$ conditions at $35{ }^{\circ} \mathrm{C}$ [36]. The average pressures were directly extracted from the MD simulations carried out at different number densities and the solubilities $S_{\text {gas }}(p)$ were obtained from TPI analyses. Both $C_{\text {gas }}(p)$ and $S_{\text {gas }}(p)$ were then fitted to analytical functions, which are provided in the Supporting Information along with the fit parameters (Table S4).

\subsection{The iterative GCMC-MD method for penetrant sorption}

Due to their periodicity in three dimensions, molecular bulk models lack explicit reservoirs, which is problematic when the uptake from a gas reservoir held at a fixed pressure $p$ has to be modelled [42]. It is possible to prepare fully-atomistic films with reservoirs on either side, but these are very expensive calculations in terms of simulation times and, furthermore, they require intricate preparation procedures to properly represent the transitions from the surface-like to the bulk-like membrane core regions [82]. As such, they are still quite rare [83-86]. On the other hand, it is possible to adjust the penetrant loading within a dense matrix using the Grand Canonical Monte Carlo (GCMC) technique [76,87]. It ensures that the number of penetrant molecules inserted into the matrix phase is in equilibrium with an external gas of pressure $p$, based on the equality of the chemical potential for the penetrant in the gas phase, $\mu_{\text {gas }}$, and that for the penetrant in the matrix phase, $\mu_{\text {matrix }}[80,88]$.

GCMC calculations are carried out under $\mu V T$ conditions, i.e. at constant chemical potential, volume and temperature. Here a virtual gas reservoir of pressure $p$ exchanges penetrant molecules with the static matrix of fixed volume $V$ using Monte Carlo moves to establish an equilibrium between both phases. There are two types of trial moves: the transfer from the gas phase to the matrix phase, which is referred to as "insertions into the matrix" and the opposite, which is referred to as "deletions from the matrix". The characteristics of the gas phase, $C_{g a s}(p)$ and $S_{\text {gas }}(p)$, are directly taken from the pure gas data (Section 3.1).

As for TPI, the efficiency of GCMC can be significantly improved [36] by using the EVMS formalism, which again screens out the dense regions of the matrix with very low insertion probabilities. In practice, the matrix phase is first scanned to identify the volume $V_{\text {in }}$ where insertions will be attempted. This defines the EVMS unbiaising factor $u_{b}$ as:

$u_{b}=\frac{V_{\text {in }}}{V}$

GCMC then selects a penetrant molecule from either the gas phase at pressure $p$ or from the matrix phase. Its probability of being moved into the other phase is related to its Boltzmann factor for the energy change upon insertion, $\exp \left(\frac{-\Delta \Phi}{k_{B} T}\right)$. For the gas phase, the Boltzmann factor simply corresponds to $S_{\text {gas }}(p)$ (Eq. (2)), but for the matrix phase, it has to be explicitly calculated. Since there are no insertions into the screenedout part (which would have essentially been associated to negligible Boltzmann factors), the probability of insertion into the matrix phase $p_{i m}$ can then be written as:

$$
p_{i m}=\frac{\exp \left(-\frac{\Delta \Phi}{k_{B} T}\right)}{S_{\text {gas }}(p)+\exp \left(-\frac{\Delta \Phi}{k_{B} T}\right)} \approx \frac{u_{b} \exp \left(-\frac{\Delta \Phi}{k_{B} T}\right)_{i n}}{S_{\text {gas }}(p)+u_{b} \exp \left(-\frac{\Delta \Phi}{k_{B} T}\right)_{i n}}
$$

Similarly, the probability of insertion into the gas phase $p_{\text {ig }}$ takes into account the bias of the EVMS formalism:

$p_{\text {ig }}=\frac{S_{\text {gas }}(p)}{S_{\text {gas }}(p)+\exp \left(-\frac{\Delta \Phi}{k_{B} T}\right)} \approx \frac{S_{\text {gas }}(p)}{S_{\text {gas }}(p)+u_{b} \exp \left(-\frac{\Delta \Phi}{k_{B} T}\right)_{\text {in }}}$ 
The proposed move is then accepted or refused depending on $p_{i g}$ and $p_{i p}$ and this process is repeated many millions of times until detailed balance is obtained, i.e. the flux of molecules from the gas phase to the matrix phase is the same as that from the matrix phase to the gas phase. For rigid penetrant molecules, convergence translates into a fairly simple relationship between $C_{g a s}, S_{\text {gas }}$ and the concentration and the solubility of the penetrant in the matrix phase, $C_{\text {matrix }}$ and $S_{\text {matrix }}$ at pressure $p$ [28,36,80,81,89]:

$\frac{C_{\text {matrix }}(p)}{C_{\text {gas }}(p)}=\frac{S_{\text {matrix }}(p)}{S_{\text {gas }}(p)}$

Convergence is deemed to be attained once the number of sorbed penetrant molecules in the matrix phase stabilizes and Eq. (7) is obeyed. However, it is important to average over several different configurations of the matrix phase since the penetrant solubilities can significantly vary from one to another. For both $\mathrm{CH}_{4}$ and $\mathrm{CO}_{2}$, the typical numbers of GCMC trial moves used here amounted to 20 million per configuration and averages were made over 20 separate configurations of the matrix phase.

GCMC is a powerful technique to predict sorption, but since the trials are done on static pre-stored matrix phase configurations, it is unable to take into account the possible volume changes and relaxation of the matrix following the change in the number of penetrants. It is thus combined with NPT MD, which allows for the system to relax following a change in the number of penetrants [45-48]. However, this relaxation perturbs the equality in Eq. (7) and GCMC has to be re-used in order to adapt the number of sorbed penetrant molecules to the newly-relaxed system. A new MD relaxation phase follows and so forth. These iterative GCMC-MD cycles have to be repeated until convergence is obtained. The number of iterative cycles is typically $\sim 5-10$ for $\mathrm{CH}_{4}$ but in general, more iterations, i.e. $\sim 5-40$ depending on $p$, are required for the highly-plasticizing $\mathrm{CO}_{2}$ where the extensive volume dilation leads to slow convergence $[28,36]$.

In the polyOAPS-imide and polyPOSS-imide networks, a $\mathrm{CH}_{4}$ or $\mathrm{CO}_{2}$ sorption curve starts with a GCMC phase on 20 representative configurations of the matrix where the target pressure of the virtual gas phase is set to 1 bar. An optimum final configuration is then chosen amongst the 20 and, if necessary, its number of penetrants is adjusted to the average obtained from all the GCMC runs. This configuration is then simulated for 200 ps with NVT MD in order to establish thermal equilibrium, before being allowed to relax under NPT conditions for $1800 \mathrm{ps}$ with a target pressure of 1 bar. The last $20 \mathrm{MD}$ configurations are then used in a second campaign of GCMC sampling. This is again followed by a second MD simulation of 200 ps under NVT conditions and 800 ps under NPT conditions. The latter phase is shortened since the number of penetrant molecules added is much less than at the first iteration. This iterative GCMC-MD process then continues until the number of sorbed penetrant molecules in the relaxed matrix phase stabilizes and the concentration ratio equals the solubility ratio (Eq. (7)). Subsequent iterative GCMCMD simulations are performed in a similar way at different pressures over the whole range considered, i.e. in the present case at 2, 5, 10, 20, 40 and 60 bar.

\section{Molecular characterization of the polyOAPS/POSS-imide hyper-cross-linked networks}

The analyses of the pure networks were all performed at $35^{\circ} \mathrm{C}$, i.e. at the same temperature than the sorption curves.

\subsection{Connectivities}

Compounds based on POSS and their phenyl derivative OAPS have a lot of potential in the field of separation membranes [17], but their multi-arm nature gives rise to very complex molecular connectivities. Three main types of organic-inorganic links can be identified [26,27,
30]. These are by decreasing order of occurrence: i) the intercage single-links, which are imides linked to two different cages, ii) the intracage links, which are imides attached to two arms of the same cage and iii) the intercage double-links, which are two imides linked to two different arms of the same two cages. The number of arms linked per cage varies from 0 to 8 and as such the average number of arms linked per cage is not sufficient to fully characterize the connectivities. In addition, the actual cross-linking densities, i.e. the number of different cages linked to a cage, are lower than the average number of linked arms per cage due to the intracage links and double intercage links. The connectivities were analysed in our screening work at high temperatures [30] and the main features for the eight networks under study are summarized in Table 1. Fig. 2a gives the probability density distributions of the number of arms linked per OAPS in all three polyOAPS-6FDA networks and Fig. 2b displays a snapshot of one of them, the polyparaOAPS-6FDA.

All these networks have an average number of links per cage of $\sim 5$, although the distributions are quite broad (Fig. 2a). In addition, the proportions of intercage and intracage links vary significantly depending on the nature of their precursors. A higher average number of intercage links is clearly an asset for the mechanical resistance, and in this respect, the PMDA networks appear better than the 6FDA networks. However, there is one exception, the polyorthoOAPS-PMDA, where the combination of the ortho geometry and the short PMDA linker makes it harder to form intercage links but leads to a higher proportion of intracage links. This is confirmed by the average characteristic distances and angles for intercage links reported in Table 2, i.e. the intercage $\mathrm{Si}$ ‥Si distances (which represent the total length of the linkers) and the imide $\mathrm{N} \cdots$ centre $\cdots \mathrm{N}$ angles (with the centres being the two middle aromatic carbons for PMDA and the $\left(\mathrm{CF}_{3}\right)_{2}$-carrying carbon for $\left.6 \mathrm{FDA}\right)$. The orthoOAPS behave significantly differently from the para and meta isomers with a tendency for its organic linker to be stretched in the case of the flexible 6FDA, whereas this is hardly possible with the rigid PMDA (Table 2)

The cross-linking density, i.e. the number of different cages linked to a cage, is $\sim 3.8$ on average and actually correlates very well with the number of intercage links. There is a rather large proportion of cages having either one intralink (which does not reinforce the network), or one intercage double-link (which reinforces the structure but counts as one for the cross-linking density). However, it is usually either one or the other, and as such their effects of one unaccounted link compensate in terms of the cross-linking density. It is also possible to get more complicated connectivities with either two intracage links or one intracage and one intercage double-link, but their occurrence is very limited because of the large steric constraints imparted on such structures.

\subsection{Densities}

The relaxed densities at $35{ }^{\circ} \mathrm{C}, \rho_{\text {network}}$, are shown in Fig. 3. The energies, which are given in Table S4 (Supp. Info), show no indications of specific molecular strains.

The density clearly depends on both types of precursors. While there are no experimental densities available yet on such thin-films, the model densities for all the precursors were tested earlier and found to be very close to their respective experimental values $[26,27,30]$. This provides confidence that cohesion is properly represented in the models. As far as the organic precursors are concerned, the flexible 6FDA leads to denser systems because of its better ability to pack. When compared to PMDA, the initial 6FDA-based model mixtures are denser, their polyPOSS-(amic-acid) intermediates are denser and there is a larger volume loss per $\mathrm{H}_{2} \mathrm{O}$ upon imidization [26,27,30], which is consistent with the experimental refractive indices [21]. On the other hand, the stiffer PMDA does not adapt well to the steric constraints introduced by cross-linking and this leads to more free volume in the corresponding networks. As far as the inorganic precursors are concerned, the $\rho_{\text {network }}$ 
Table 1

Detailed connectivities of the polyOAPS-imide and polyPOSS-imide network models.

\begin{tabular}{|c|c|c|c|c|c|c|c|c|}
\hline Connectivities & $\begin{array}{l}\text { meta OAPS } \\
+ \text { PMDA }\end{array}$ & $\begin{array}{l}\text { para OAPS }+ \\
\text { PMDA }\end{array}$ & $\begin{array}{l}\text { ortho OAPS } \\
+ \text { PMDA }\end{array}$ & $\begin{array}{l}\text { POSS }+ \\
\text { PMDA }\end{array}$ & $\begin{array}{l}\text { meta OAPS }+ \\
6 \text { FDA }\end{array}$ & $\begin{array}{l}\text { para OAPS }+ \\
6 \text { FDA }\end{array}$ & $\begin{array}{l}\text { ortho OAPS }+ \\
\text { 6FDA }\end{array}$ & $\begin{array}{l}\text { POSS }+ \\
6 \text { FDA }\end{array}$ \\
\hline Total no. of atoms & 33672 & 33828 & 33492 & 31644 & 43183 & 43245 & 41974 & 41362 \\
\hline$<$ No. of links per cage $>$ & 5.3 & 5.4 & 5.2 & 5.1 & 4.9 & 4.9 & 4.5 & 4.9 \\
\hline$<$ No. of intercage links per cage $>$ & 4.6 & 5.1 & 3.3 & 4.3 & 3.7 & 3.7 & 3.9 & 4.1 \\
\hline$<$ No. of intracage links per cage $>$ & 0.7 & 0.3 & 1.9 & 0.8 & 1.2 & 1.2 & 0.6 & 0.8 \\
\hline $\begin{array}{l}<\text { No. of different cages linked to a cage }>(= \\
\text { cross-linking density })\end{array}$ & 4.2 & 4.7 & 3.0 & 4.0 & 3.4 & 3.5 & 3.6 & 3.7 \\
\hline$<\%$ of cages having only intercage single-links $>$ & 36.6 & 54.6 & 19.9 & 42.6 & 31.0 & 37.0 & 49.1 & 42.1 \\
\hline $\begin{array}{l}<\% \text { of cages having one intracage link }+ \text { all } \\
\text { others intercage single-links }>\end{array}$ & 31.5 & 13.9 & 49.5 & 30.1 & 39.8 & 38.9 & 27.3 & 28.7 \\
\hline $\begin{array}{l}<\% \text { of cages having one intercage double-link }+ \\
\text { all others intercage single-links }>\end{array}$ & 26.8 & 24.5 & 7.9 & 19.4 & 15.7 & 13.4 & 16.7 & 19.4 \\
\hline
\end{tabular}

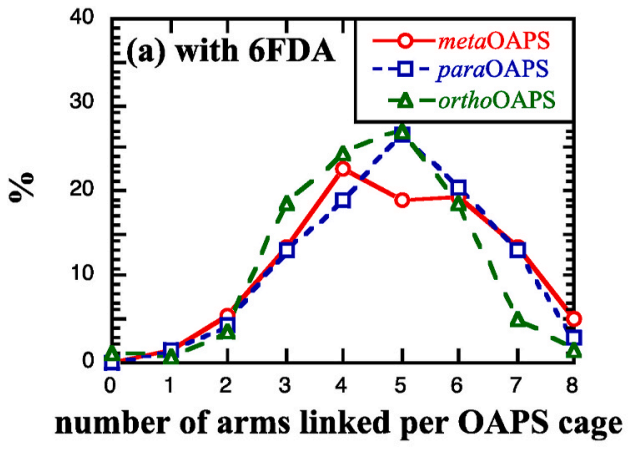

(b)

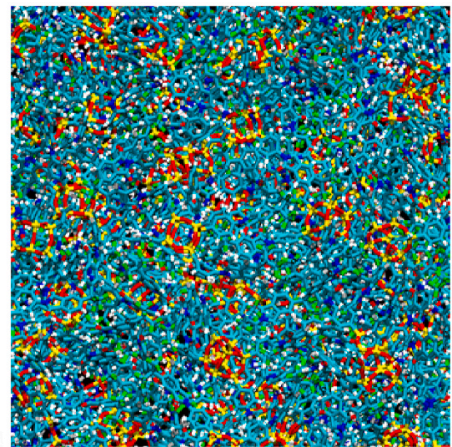

Fig. 2. (a) Percentages of OAPS cages as a function of their number of arms linked in the three polyOAPS-6FDA networks. (b) Schematic representation of a (50 $\AA^{2}$ subset of the polyparaOAPS-6FDA network (colour code: yellow $=\mathrm{Si}$, red $=\mathrm{O}$, cyan $=\mathrm{C}$, blue $=\mathrm{N}$, green $=\mathrm{F}$, white $=\mathrm{H}$ ). (For interpretation of the references to colour in this figure legend, the reader is referred to the Web version of this article.)

Table 2

Average intercage $\mathrm{Si} \cdots \mathrm{Si}$ distances and imide $\mathrm{N} \cdots$ centre $\cdots \mathrm{N}$ angles.

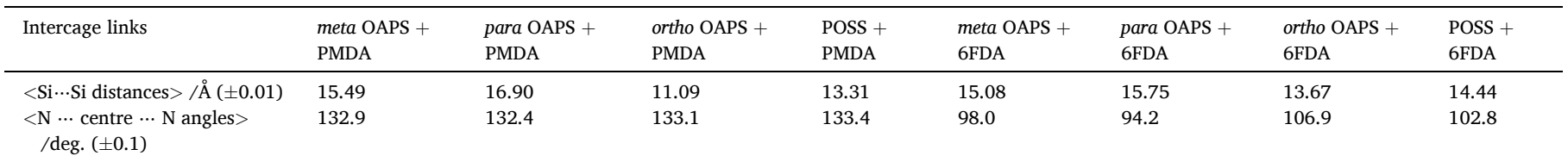
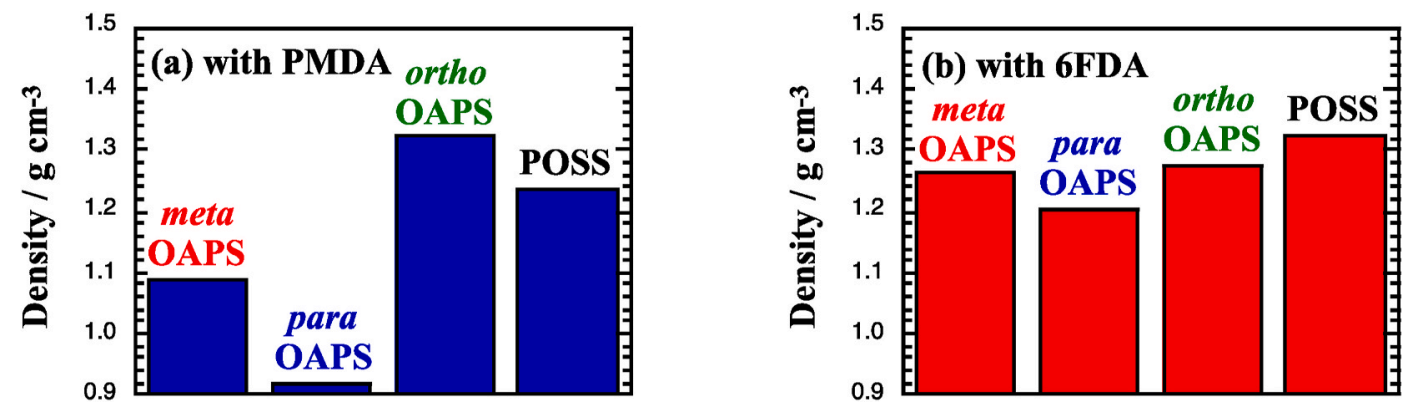

Fig. 3. Compared average densities $\rho_{\text {network }}$ for the polyOAPS-imides and polyPOSS-imides. The maximum standard error is $0.001 \mathrm{~g} \mathrm{~cm}^{-3}$. 
vary in the order paraOAPS $<$ metaOAPS $<$ orthoOAPS. This can be related to their molecular dimensions in the pure state, i.e. an average length (defined as twice the average distance between the centre of a siloxane cage and its eight primary amine $\mathrm{N}$ ) of $\sim 17.4 \AA$ for the para, $\sim 15.5 \AA$ for the meta and $\sim 10.2 \AA$ for the ortho isomers, respectively. Interestingly, the differences are very much enhanced when associated to the stiff PMDA precursor. This results in polyorthoOAPS-PMDA being a much denser structure than its other isomers as it combines the constraints of both its inorganic and organic precursors. It is also denser than the polyPOSS-PMDA as its average $\mathrm{Si}$...Si distance is shorter (see Table 2).

\subsection{Free-volume morphology}

In molecular simulations of dense matrices, the free-volume or voidspace can be characterized using different techniques [23,43,90,91]. A simple geometric approach is the percentage of probe-accessible volume, \%PAV, which is obtained by repeated random trial insertions of a virtual spherical probe of predefined radius [92]. The insertions are only successful when the probe does not overlap with the matrix atoms, represented in this particular analysis as hard spheres with standard van der Waals radii [93]. In the present case, up to 20 insertions of a probe of radius $1.784 \AA$ (half the $\mathrm{N}_{2}$ kinetic diameter [94]) were attempted for each $\AA^{3}$ of the 400 configurations stored in the production runs. The average $\% P A V$ thus characterizes the fraction of void-space that can accommodate such a spherical probe without any assumptions on the forms of the holes and without any energetic considerations. Smaller probe sizes such as He can be used too, but they can insert into very small holes, where there are hardly any chances of getting any other penetrants. As such, half the $\mathrm{N}_{2}$ kinetic diameter is a good compromise between avoiding those holes that are too small and still being able to sample the more significant ones. The $<\% P A V_{0}>$ (with the subscript "0" referring to the pure matrices) are displayed in Fig. 4, and as for the other properties, they vary depending on both types of precursors. They generally correlate negatively with the densities (Fig. 3) and confirm that the phenyl-arm OAPS isomers result in much more open structures than the aliphatic-arm POSS. The maximum amount of accessible void-space is reached for polyparaOAPS-PMDA, which combines the linear arms of the para isomer with the linear geometry of the stiff PMDA linker. However, both the other two OAPS isomers also create more space than the flexible $-\left(\mathrm{CH}_{3}\right)-\mathrm{NH}_{2}$ POSS linkers because of the intrinsic planarity and rigidity of their phenyl rings. Once again, the differences are enhanced when associated to the PMDA organic linker, as it only allows limited relaxation of the network upon imidization [27]. On the other hand, the ability of the 6FDA angle to stretch, which is especially noticeable in the case of the polyorthoOAPS-6FDA (Table 2), somewhat relaxes the constraints of the inorganic precursor.

A more elaborate assessment is provided by the TPI method (Section 3.1.), which accounts for both the geometric and energetic dependence of the penetrant solubility in the pure matrices. The Boltzmann- weighted probability density distributions $\rho_{w}(\Delta \Phi)$ (Eq. (3)) upon insertion of realistic $\mathrm{CH}_{4}$ or $\mathrm{CO}_{2}$ model probes [53,54] support the Gaussian site-distribution model $[95,96]$ and provide the range of site energies for penetrant adsorption $[27,83,90]$. The lower $\Delta \Phi$ are associated to the most favourable and the higher $\Delta \Phi$ to the least favourable sites for insertion. A comparison of the $\rho_{w}(\Delta \Phi)$ for both $\mathrm{CH}_{4}$ and $\mathrm{CO}_{2}$ in the polymetaOAPS-PMDA network (Fig. 5a) shows their large difference in infinite-dilution solubility $S_{\text {matrix } 0}$, which is obtained from the area under these curves [28]. Fig. 5b-c compares the $\rho_{w}(\Delta \Phi)$ for $\mathrm{CH}_{4}$ and $\mathrm{CO}_{2}$ in all PMDA networks. The $\rho_{w}(\Delta \Phi)$ for the 6FDA networks are rather similar on the same scales (Fig. $5 \mathrm{~d}-\mathrm{e}$ ), which suggests that the free-volume morphology is mostly governed by the nature of the inorganic precursor, although the influence of the organic linker is more noticeable in certain cases, e.g. the paraOAPS with $\mathrm{CO}_{2}$.

All the networks exhibit fairly homogeneous Gaussian-like distributions over a range of high-energy to low-energy sites, which are displaced towards the lower $\Delta \Phi$ for $\mathrm{CO}_{2}$. The largest standard deviations are found for the paraOAPS isomer, where the low density is associated to a large variety of hole morphologies. However, the integrals under the $\rho_{w}(\Delta \Phi)$ curves, i.e. the $S_{\text {matrix } 0 \text {, are almost as high in the metaOAPS }}$ networks, as opposed to the orthoOAPS and POSS networks. The most energetically-favourable sites for the insertion of $\mathrm{CO}_{2}$ (corresponding to $\left.\Delta \Phi / k_{B} T<-15\right)$ are superimposed with the siloxane cages and the inorganic arms in Fig. 6 (low energy sites in yellow, cages in red, inorganic arms in green, primary amine or imide $\mathrm{N}$ in blue). The rest of the organic imides and the hydrogens are omitted from Fig. 6 and the images are clipped on thicknesses of a few $\AA$ to better visualize the positions of the lowest energy sites with respect to the inorganic phase.

The lowest-energy sites are well-spread in both the paraOAPS and the metaOAPS networks. In these matrices, most of them are close to the siloxane cage oxygens, i.e. they are found in the spaces between the cross-linked and/or un-cross-linked OAPS arms (Fig. 6a-b). There are even extended clusters of very low energy sites (Fig. 6b). On the other hand, the orthoOAPS geometry prevents access to the cages, its lowenergy sites are less numerous and tend to be closer to the arms of the inorganic precursor but further away from the cages (Fig. 6c). For POSS, the lowest-energy sites are mostly spread in the organic phase (Fig. 6d). The various $\rho_{w}(\Delta \Phi)$ result in significantly-different infinite-dilution solubilities $S_{\text {matrixo }}$. Their relative values with respect to those of the highest-solubility networks $S_{\text {matrix0-max }}$ i.e. the polymetaOAPS-PMDA for $\mathrm{CH}_{4}$ and the polyparaOAPS-PMDA for $\mathrm{CO}_{2}$, are provided in Table 3 . The orthoOAPS and POSS $S_{\text {matrix } 0}$ are indeed roughly half of those in the paraOAPS and metaOAPS networks. As noted from Fig. 5, the organic PMDA or 6FDA linker has much less of an influence on the $S_{\text {matrixo. }}$

A related property is the fraction of significant volume (FSV), which is the part of the matrix actually responsible for the penetrant solubility, and can be estimated from the unweighted $\rho(\Delta \Phi)$ and weighted $\rho_{w}(\Delta \Phi)$ distributions [52]. To account for $100 \%$ of the solubility would mean considering all energies from $-\infty$ to $+\infty$. However, Fig. 5 shows clearly that the integrals of the weighted functions will reach a plateau (a) with PMDA

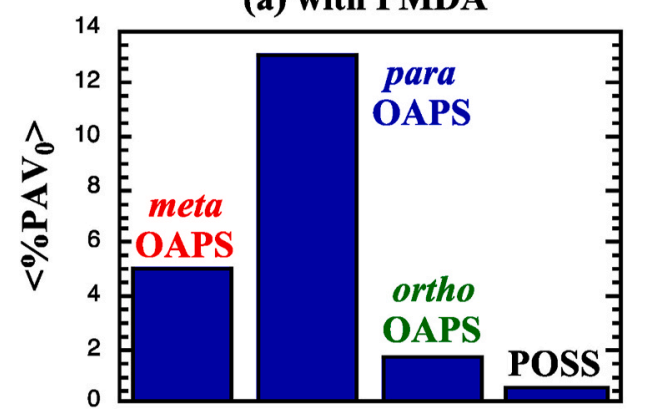

b) with 6FDA

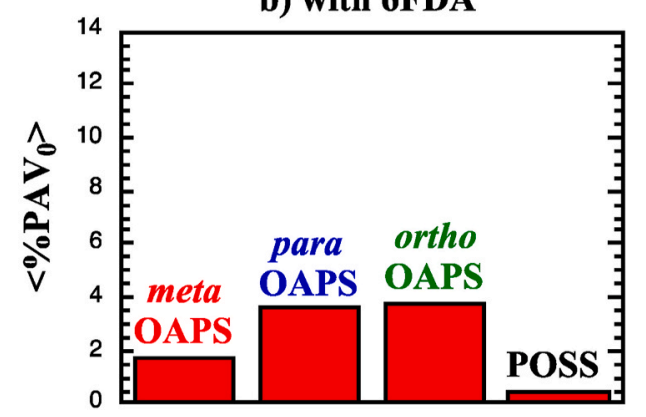

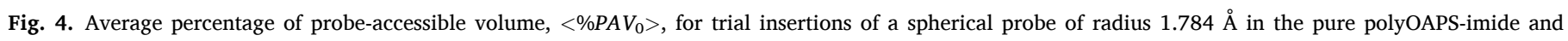
polyPOSS-imide networks. The maximum standard error is $0.02 \%$. 


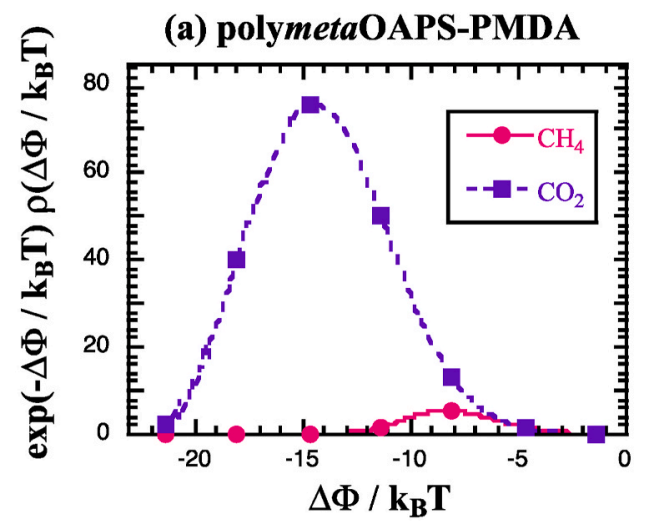

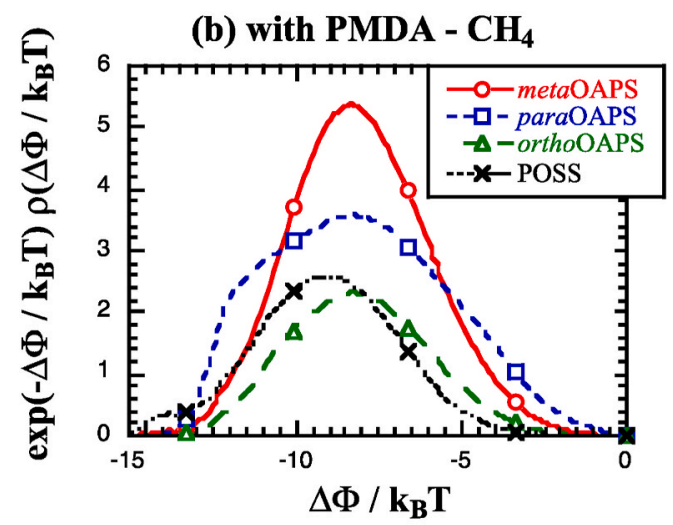

(d) with 6FDA - $\mathrm{CH}_{4}$

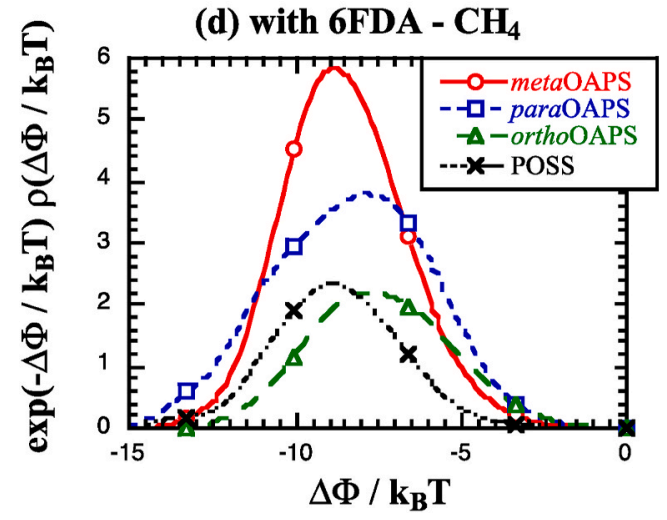

(c) with PMDA - $\mathrm{CO}_{2}$
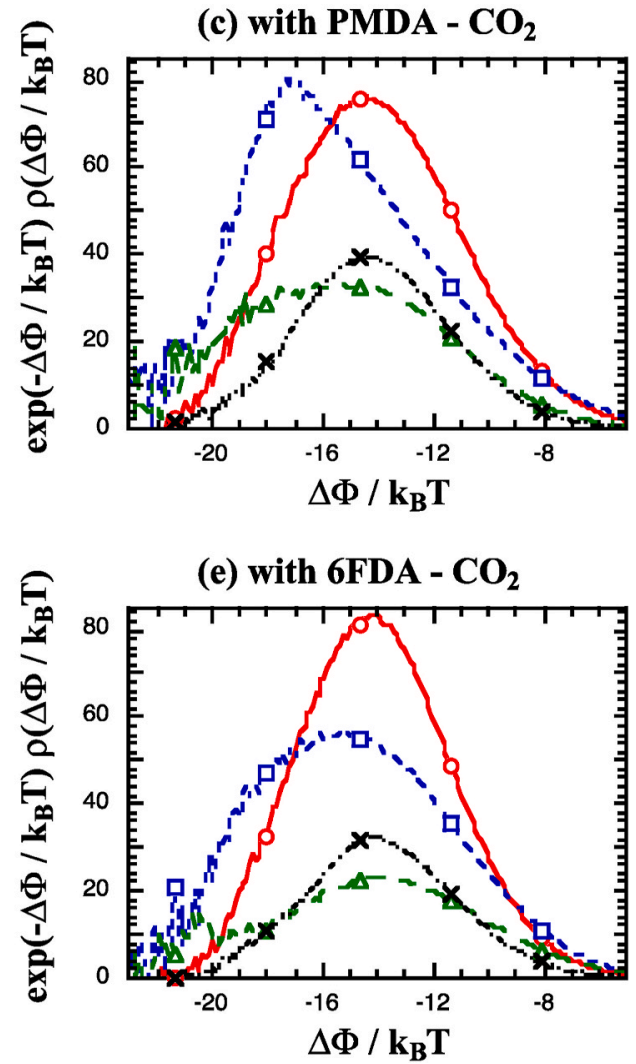

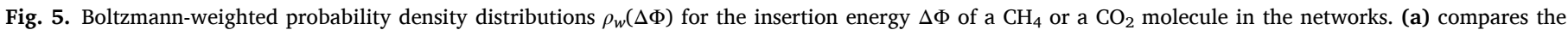

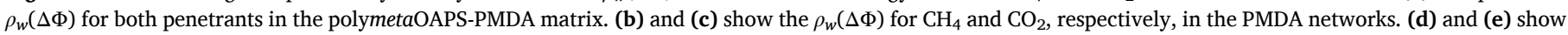
the corresponding data for the 6FDA networks.

relatively quickly as the insertion energy increases. As such, it is possible to define a critical upper limit of the integral over $\rho_{w}(\Delta \Phi)$ that accounts for $99.9 \%$ of the solubility, and which we refer to as $\Delta \Phi_{\mathrm{c}}$. The $F S V$ is the fraction of random insertions that lead to energies in the range from $-\infty$ to $\Delta \Phi_{\mathrm{c}}$ and thus contribute in practice to $99.9 \%$ of the solubility. It is given by the integral of the normalized unweighted probability density $\rho(\Delta \Phi)$ up to the critical upper limit $\Delta \Phi_{\mathrm{c}}$ (Eq. (8)):

$F S V=\int_{-\infty}^{\Delta \Phi_{c}} \rho(\Delta \Phi) d \Delta \Phi$

The $<\% F S V_{0}>$ for both penetrants in the pure matrices are reported in Table 3 along with the relative infinite-dilution solubilities. For methane, the $\angle \% F S V_{0}>$ are close to the $\angle \% P A V_{0}>$ obtained from the geometric insertion of a spherical probe (Fig. 4). This suggests that the solubility of $\mathrm{CH}_{4}$ mostly comes from the available void-space in the matrix. For the more soluble $\mathrm{CO}_{2}$, the insertion energy distributions are displaced towards much lower energies (Fig. 5a). Only those sites that provide favourable interactions give a significant contribution to the solubility, thus the fraction of useful volume for insertion in the matrix, $<\% F S V_{0}>$ for $\mathrm{CO}_{2}$, decreases. However, the relative order remains the same as that of the $\left.\angle \% P A V_{0}\right\rangle$ and the methane $\left\langle \% F S V_{0}\right\rangle$, which confirms the importance of the amount of available void-space in the pure matrix in addition to the interactions.

\section{Single-gas sorption isotherms for $\mathrm{CH}_{4}$ and $\mathrm{CO}_{2}$ in the polyOAPS/POSS-imide networks}

The $\mathrm{CH}_{4}$ and $\mathrm{CO}_{2}$ sorption isotherms in the networks under study were obtained at $35{ }^{\circ} \mathrm{C}$ and over the full $0-60$ bar range by using the iterative GCMC-MD technique. As mentioned in Section 3.2., it is important to average the GCMC sorption phase over enough configurations and to allow the MD relaxation phase to properly sample the 
(a) polymetaOAPS-PMDA

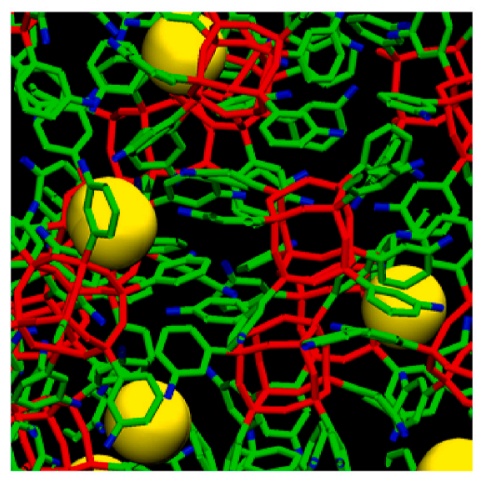

(c) polyorthoOAPS-PMDA

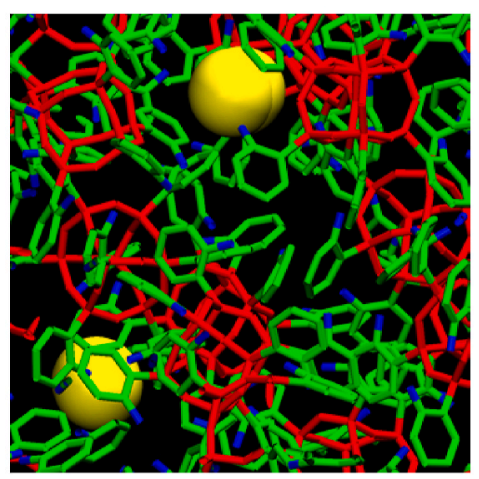

(b) polyparaOAPS-PMDA

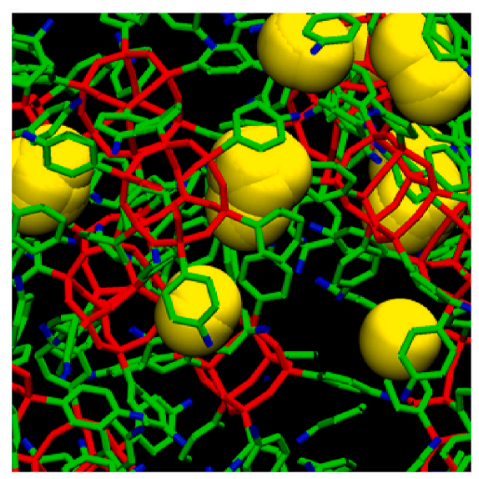

(d) polyPOSS-PMDA

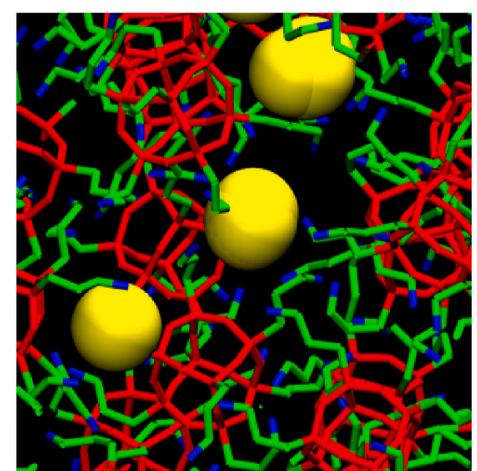

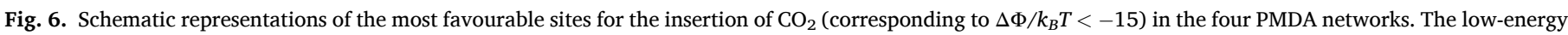

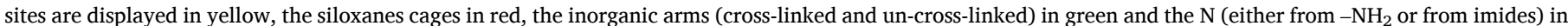
blue. (For interpretation of the references to colour in this figure legend, the reader is referred to the Web version of this article.)

Table 3

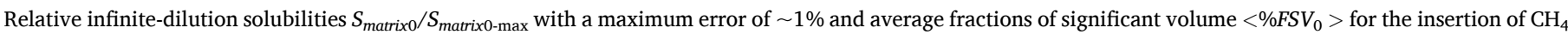
and $\mathrm{CO}_{2}$ in the pure matrices.

\begin{tabular}{|c|c|c|c|c|c|c|c|c|}
\hline & $\begin{array}{l}\text { meta OAPS }+ \\
\text { PMDA }\end{array}$ & $\begin{array}{l}\text { para OAPS }+ \\
\text { PMDA }\end{array}$ & $\begin{array}{l}\text { ortho OAPS }+ \\
\text { PMDA }\end{array}$ & $\begin{array}{l}\text { POSS + } \\
\text { PMDA }\end{array}$ & $\begin{array}{l}\text { meta OAPS + } \\
6 \text { FDA }\end{array}$ & $\begin{array}{l}\text { para OAPS }+ \\
6 \text { FDA }\end{array}$ & $\begin{array}{l}\text { ortho OAPS + } \\
6 \text { FDA }\end{array}$ & $\begin{array}{l}\text { POSS }+ \\
\text { 6FDA }\end{array}$ \\
\hline$S_{\text {matrix } 0}$ for $\mathrm{CH}_{4}$ & $100 \%$ & $94 \%$ & $45 \%$ & $50 \%$ & $95 \%$ & $86 \%$ & $43 \%$ & $40 \%$ \\
\hline$\frac{S_{\text {matrix } 0 \text {-max }}}{S_{\text {matrix } 0}}$ for $\mathrm{CO}_{2}$ & $93 \%$ & $100 \%$ & $51 \%$ & $42 \%$ & $89 \%$ & $82 \%$ & $34 \%$ & $33 \%$ \\
\hline$<\% F S V_{0}>$ for $\mathrm{CH}_{4}$ & 7.5 & 16.4 & 2.8 & 1.2 & 3.3 & 5.8 & 5.5 & 1.0 \\
\hline$<\% F S V_{0}>$ for $\mathrm{CO}_{2}$ & 4.4 & 8.5 & 1.6 & 0.8 & 2.0 & 3.5 & 3.4 & 0.6 \\
\hline
\end{tabular}

(a) polyparaOAPS-6FDA - $\mathrm{CO}_{2}$

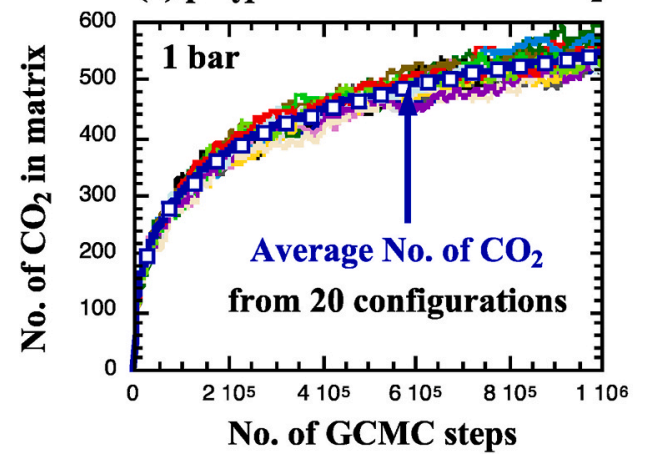

(b) polyorthoOAPS-PMDA - $\mathrm{CO}_{2}$

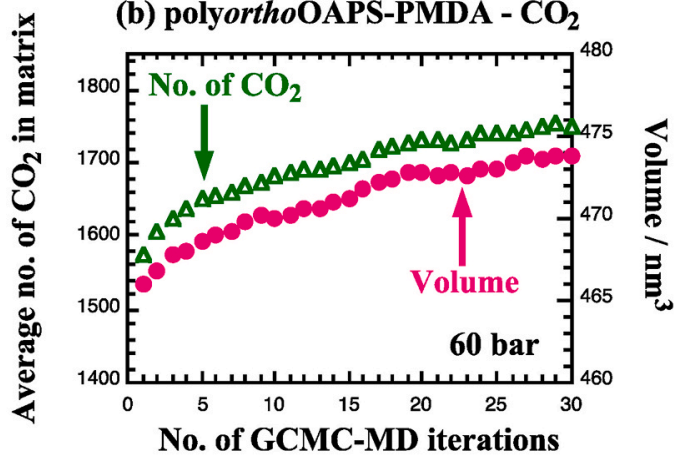

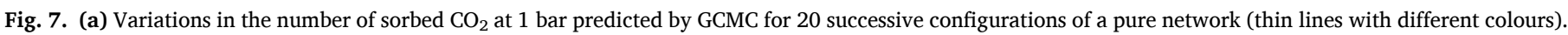

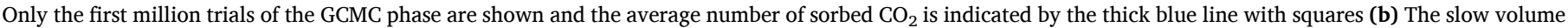

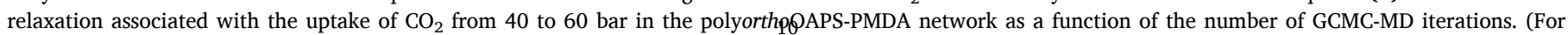
interpretation of the references to colour in this figure legend, the reader is referred to the Web version of this article.) 
slow changes in the matrices [36]. As for other glassy materials [83,84, 86,97 ], the network mean-square displacements at $35^{\circ} \mathrm{C}$ are typically of the order of $1-2 \AA^{2}$ over time-intervals of $1 \mathrm{~ns}$. Relaxations thus only occur on a local scale, and furthermore, they are very slow on the timescale of MD simulations [28,85].

Fig. 7a illustrates the variations in the number of sorbed $\mathrm{CO}_{2}$ predicted by GCMC for a gas reservoir pressure of 1 bar over just one million trials for 20 successive stored configurations (separated by 5 ps intervals) of the pure polyparaOAPS-6FDA network. Although this only represents the start of a full 20-million-trial GCMC phase, the number of sorbed $\mathrm{CO}_{2}$ molecules between specific configurations can already differ from the average by $\sim \pm 50$, i.e. $\sim \pm 10 \%$ of the average number sorbed. Averaging over a sufficient number of configurations thus strongly reduces this source of error.

Fig. 7b shows the number of successive GCMC-MD iterations needed for the volume to stabilize when the polyorthoOAPS-PMDA network at 40 bar of $\mathrm{CO}_{2}$ is further loaded up to 60 bar. The gradual volume swelling upon $\mathrm{CO}_{2}$ sorption leads to a rise in the solubility and consequently, the number of sorbed $\mathrm{CO}_{2}$ has to be regularly re-adjusted with GCMC, re-relaxed with MD and so on. Several combinations for the number of iterations and the length of the MD phase have been proposed $[36,45-48,50]$. In all cases, the most critical point is to take into account as much as possible the slow matrix relaxation of the system under study, especially when strongly-plasticizing penetrants such as $\mathrm{CO}_{2}$ are considered.

The actual uptake curves showing the penetrant concentration in the matrix $C_{\text {matrix }}(p)$ as a function of the gas pressure $p$ are provided in Fig. $8 \mathrm{a}-\mathrm{b}$ for $\mathrm{CH}_{4}$ and in Fig. $8 \mathrm{c}-\mathrm{d}$ for $\mathrm{CO}_{2}$. The same data are presented as a function of the gas fugacity in Fig. S5 of the Supp. Info. Both pressure or fugacity representations can be used [98,99], the curves are similar and the relative order remains the same. The matrices, which all contain between 30000 and 45000 atoms (Table 1), are large enough to provide significant statistics on the uptakes [36]. Indeed, the maximum standard errors on the $C_{\text {matrix }}(p)$, which were estimated using the method given in Ref. [87], are only $0.3 \mathrm{~cm}^{3}(\mathrm{STP}) / \mathrm{cm}^{3}$ for $\mathrm{CH}_{4}$ and 0.7 $\mathrm{cm}^{3}$ (STP) $/ \mathrm{cm}^{3}$ for $\mathrm{CO}_{2}$. As such, they are smaller than the symbols on the scale of Fig. 8.

The $C_{\text {matrix }}(p) v s p$ curves are Type I adsorption isotherms [100], and can be fitted over the entire pressure range to the dual-mode sorption (DMS) model [101]. The DMS formalism and the fit parameters corresponding to Fig. 8 are provided in the Supp. Information (Table S6). As expected from their critical temperatures [102], $\mathrm{CO}_{2}$ sorbs more than $\mathrm{CH}_{4}$, but the concentrations in the polyOAPS-imides also depend on both their inorganic and organic precursors. Interestingly, the $C_{\text {matrix }}(p)$ for the polyPOSS-PMDA and polyPOSS-6FDA are almost superimposable for a specific penetrant. This has already been investigated for a lower cross-link density [28]. When compared to polyPOSS-PMDA, the polyPOSS-6FDA matrix sorbs more penetrant molecules because of its greater proportion of organic phase, but it is compensated for in the $C_{\text {matrix }}(p)$ by the difference in the volumes. This is not the case for the polyOAPS-imide networks, where the sorption varies in the order paraOAPS $>$ metaOAPS $>$ orthoOAPS and PMDA $>$ 6FDA (except when associated to the orthoOAPS isomer). All of them have a higher sorption capacity than the aliphatic-arm POSS as suggested by the free-volume analyses (Section 4.3.). There again, the differences are enhanced for the PMDA networks, with a clear distinction between the high-solubility paraOAPS/metaOAPS on one side and the lower-solubility orthoOAP$\mathrm{S} / \mathrm{POSS}$ on the other side. The trends in sorption thus directly reflect the morphology of the pure networks (Figs. 5 and 6 and Table 3 ). In the case of the 6FDA networks, the $\angle \% P A V_{0}>$ and $\angle \% F S V_{0}>$ for the orthoOAPS are much higher than for the POSS (Fig. 4 and Table 3), and this brings the orthoOAPS uptake curves closer to the other two isomers.

Although it is difficult to measure small volume changes in experimental samples [103-106], the dilations of the matrices upon sorption can be easily monitored in molecular models. Fig. 9 displays the volume swelling of each network as a function of $C_{\text {matrix }}(p)$ for both its $\mathrm{CH}_{4}$ and
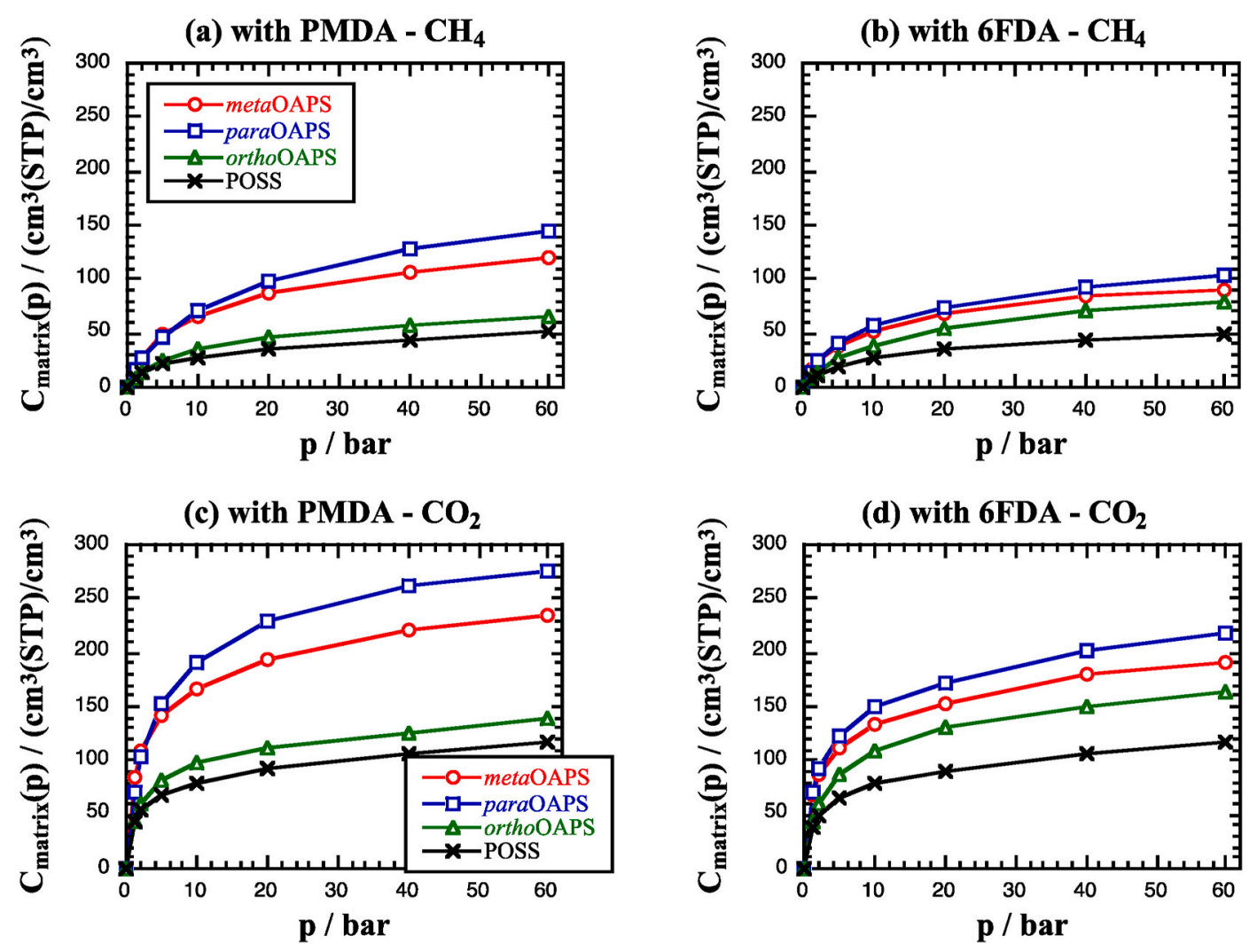

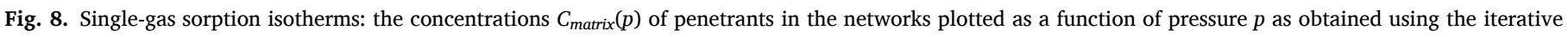
GCMC-MD method for (a-b) $\mathrm{CH}_{4}$ and (c-d) $\mathrm{CO}_{2}$. In all cases, the standard errors are less than the size of the symbols. 
(a) with PMDA - $\mathrm{CH}_{4}$

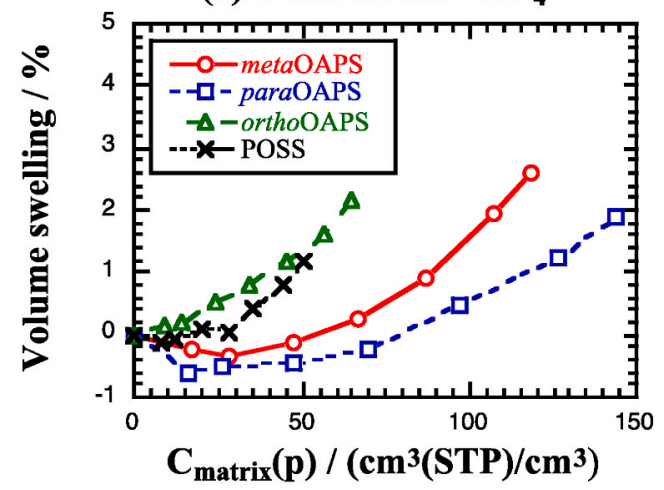

c) with PMDA - $\mathrm{CO}_{2}$

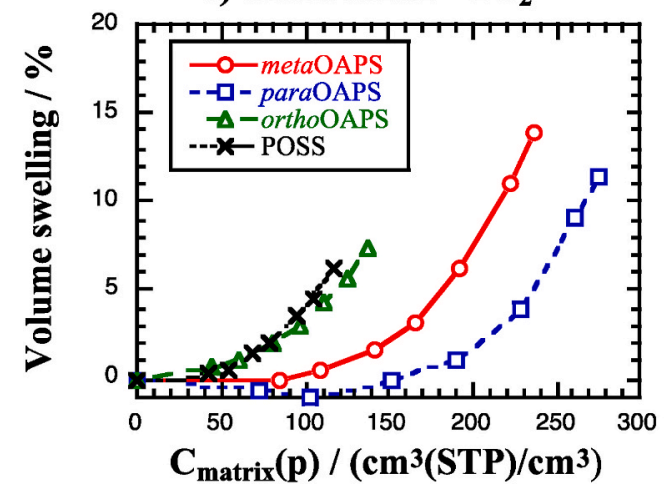

(b) with 6FDA - $\mathrm{CH}_{4}$

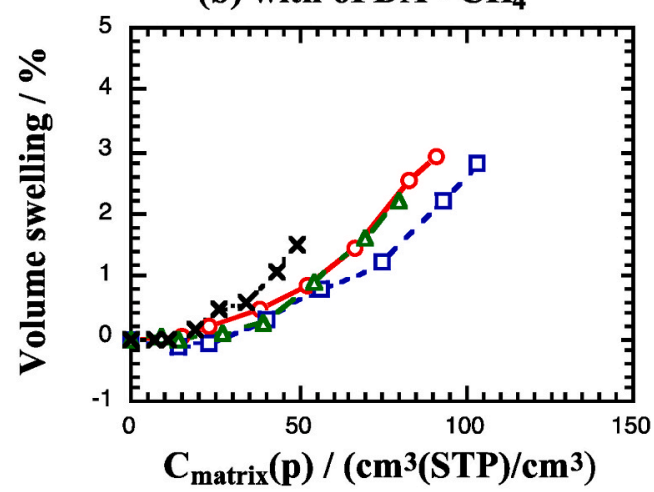

(d) with 6FDA - $\mathrm{CO}_{2}$

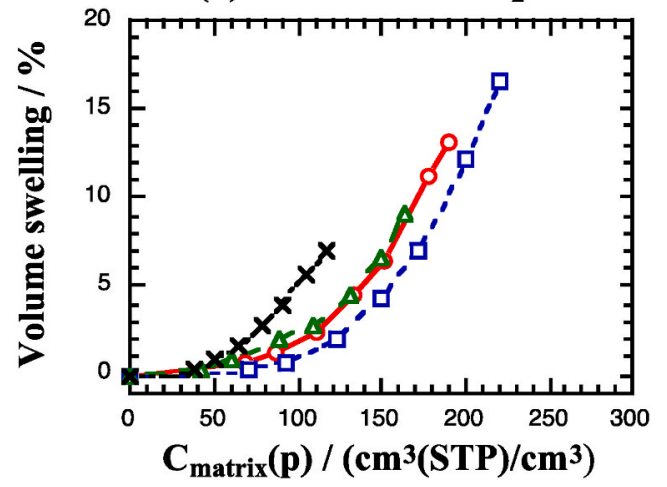

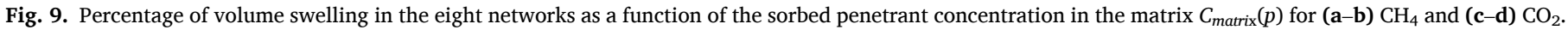
Please note that, unlike Fig. 8, the scales used for $\mathrm{CO}_{2}$ are different from those for $\mathrm{CH}_{4}$.
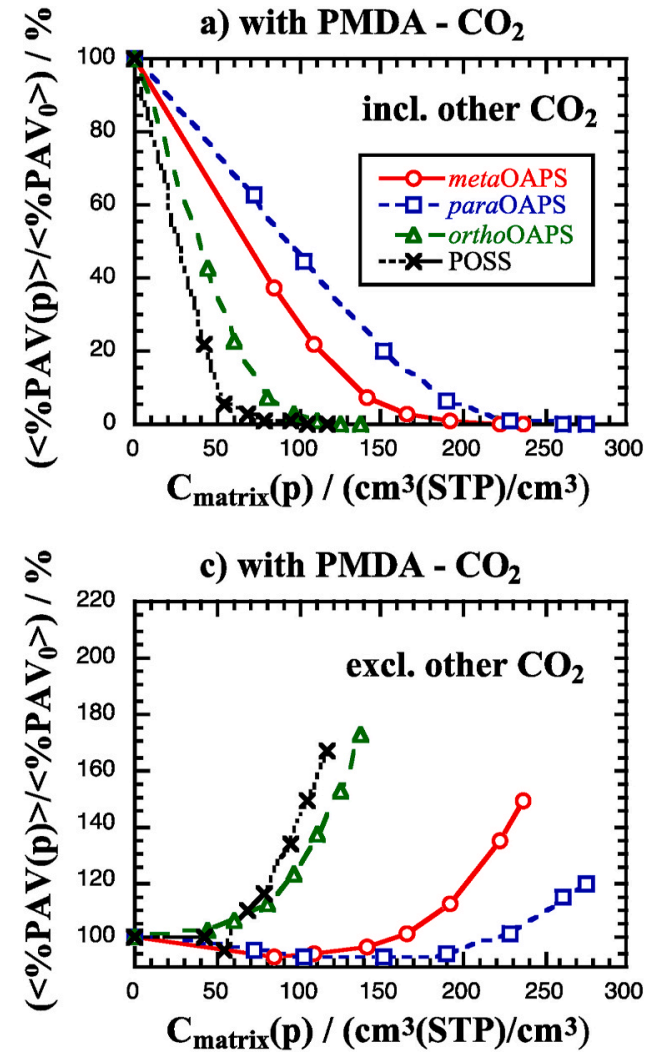
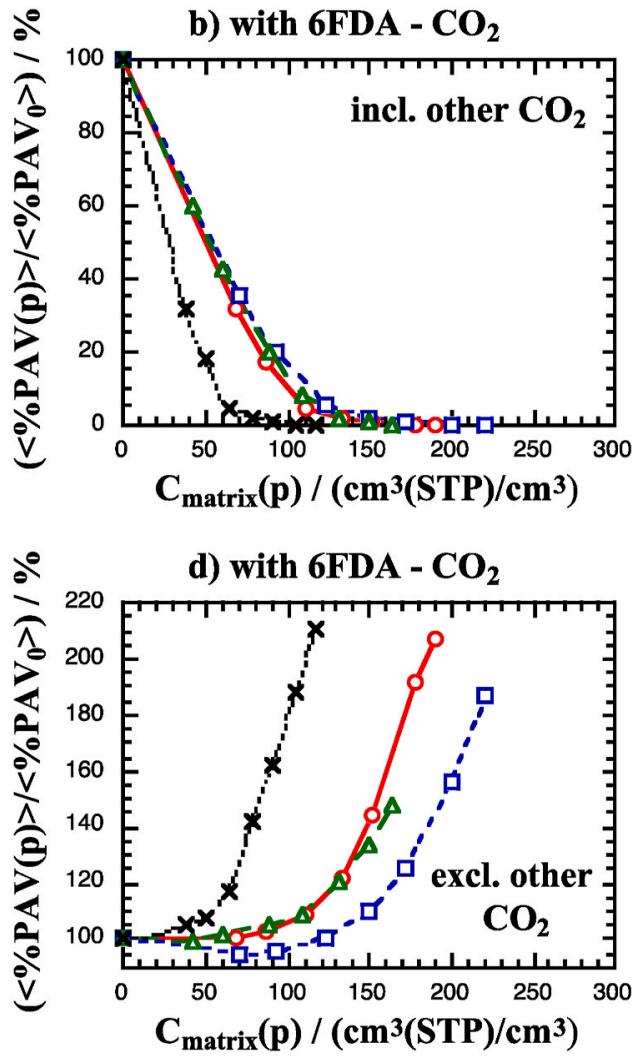

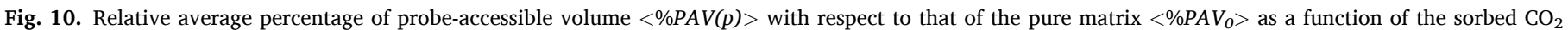
concentration in the matrix $C_{\text {matrix }}(p)$ while (a-b) including and (c-d) excluding the other $\mathrm{CO}_{2}$ present in the matrix. 
$\mathrm{CO}_{2}$ uptake curves.

The volume swelling curves in Fig. 9a-b are consistent with the mildly-plasticizing nature of $\mathrm{CH}_{4}$, which usually leads to maximum dilations of $2-3 \%$ in glassy polymers on that pressure range [81]. On the other hand, $\mathrm{CO}_{2}$ is a highly-plasticizing penetrant and as shown by Fig. $9 \mathrm{c}-\mathrm{d}$, it is able to lead to dilations of up to $10-20 \%$ at the highest pressures under study $[36,97]$. The average partial molar volumes $(p m v)$ estimated from the limiting slopes of the volume $v s$ number of sorbed molecules are $\sim 26 \mathrm{~cm}^{3} \mathrm{~mol}^{-1}$ for $\mathrm{CO}_{2}$ and $\sim 14 \mathrm{~cm}^{3} \mathrm{~mol}^{-1}$ for $\mathrm{CH}_{4}$, in agreement with the experimental $p m v$ in polyimides [103,105,107]. However, Fig. 9 clearly shows that the networks display different resistances to plasticization when compared at equivalent $C_{\text {matrix }}(p)$. The metaOAPS and paraOAPS isomers with PMDA swell less as a function of penetrant concentration due to their lower densities (Fig. 3) and associated higher free volumes (Fig. 4 and Table 3). There is even some evidence of a slight contraction at moderate $p$ due to the amount of unrelaxed free-volume in these systems [27] and the extra cohesion energy provided by the sorption of penetrants. In the case of 6FDA, a similar, albeit less pronounced, retarded swelling behaviour can be seen for OAPS, which again correlates well with their densities and free volumes. As for the uptake curves (Fig. 8), the orthoOAPS behaves similarly to POSS when associated to PMDA, and closer to metaOAPS when associated to 6FDA. However, in general, the swelling at the same $C_{\text {matrix }}(p)$ varies in the order paraOAPS $<$ metaOAPS $<$ orthoOAPS $<$ POSS. This reduced amount of swelling is an asset for the OAPS precursor as it offsets its larger sorption capacities, thus limiting the decrease in selectivities [108,109].

To relate the total swelling (Fig. 9) to that of the actual free-volume, the average probe-accessible volume $\langle \% P A V>$ was analysed for all pressures $p$ as for the $<\% P A V_{0}>$ of the pure matrices (Fig. 4), but under two conditions: (i) by including or (ii) by excluding all the other penetrant molecules in the system. Condition (i) follows the filling-up of the accessible free-volume, while condition (ii) probes its relative swelling. The decrease (upon filling-up) and increase (upon swelling) of the $<\%$ $P A V(p)>$ with respect to the $<\% P A V_{0}>$ of the pure matrices are reported in Fig. 10 as a function of the sorbed $\mathrm{CO}_{2}$ concentrations. The trends were similar for methane.

Fig. 10a-b shows the decrease in $<\% P A V(p)>$ as $C_{\text {matrix }}(p)$ increases

\section{(a) close to the siloxane cages}

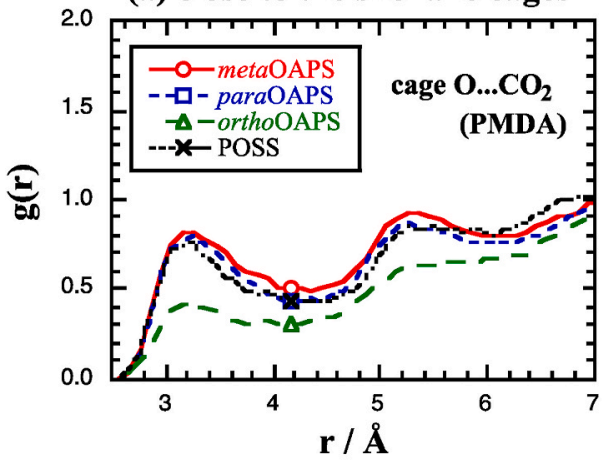

(c) close to the unreacted $\mathrm{NH}_{2}$

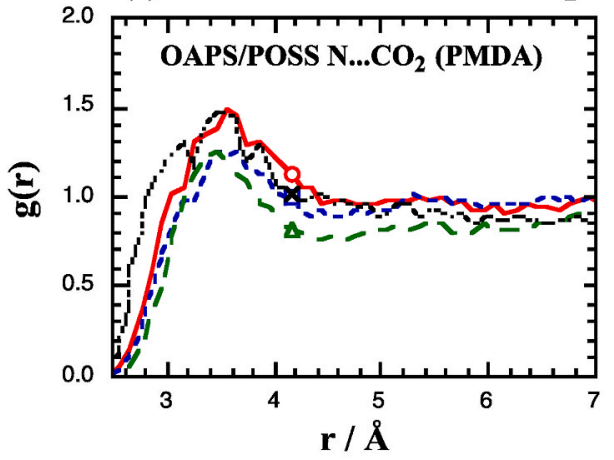

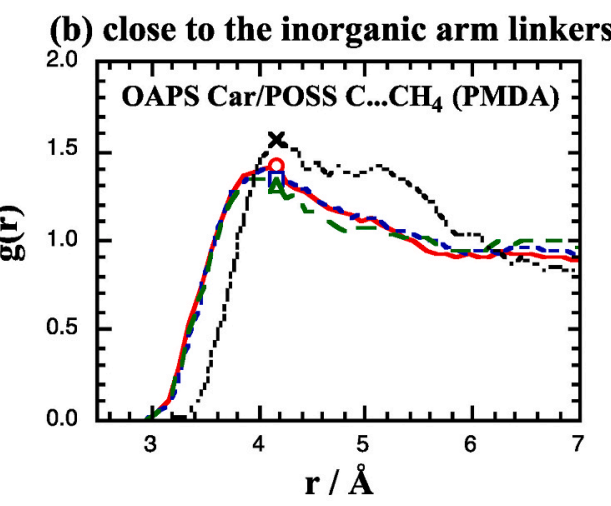

(d) close to the imide ketones

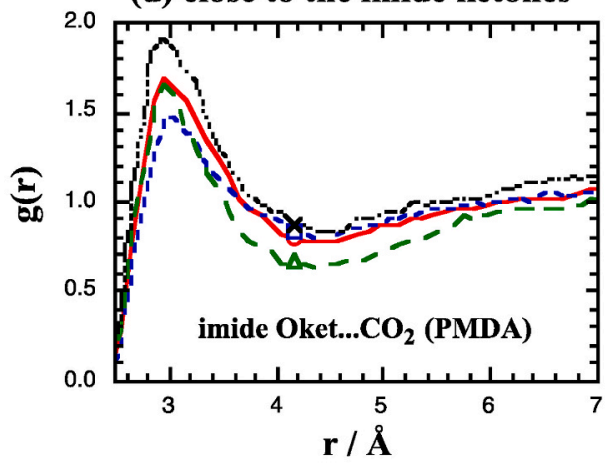

(e) close to the imide $F$

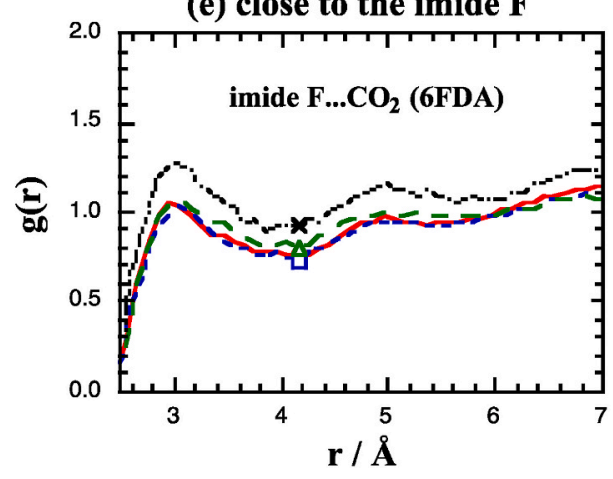

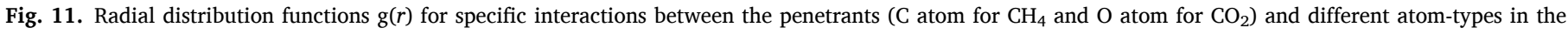
networks. The legend is the same for all plots. 
and confirm the retarded swelling of the OAPS networks due to part of their free-volume still being accessible. As noted above, the $<\% P A V_{0}>$ and $\left\langle \% F S V_{O}>\right.$ for the three isomers are closer when associated to 6FDA than to PMDA, which explains the similarity of the OAPS curves in Fig. 10b. The swelling of the free-volumes (Fig. 10c-d) also correlates with the $<\% P A V_{0}>$. In the PMDA networks, both the orthoOAPS and POSS are filled up rapidly and have to start swelling at low $C_{\text {matrix }}(p)$ to accommodate more penetrants. On the other hand, the metaOAPS and paraOAPS still have free-volume available, and as such, the volume swelling only appears at much higher $C_{\text {matrix }}(p)$. In the 6FDA networks, the situation is similar for POSS, but not for the three OAPS isomers. The lowest $<\% P A V_{0}>$ is found in metaOAPS, and its free-volume is able to swell by over $200 \%$ at the highest penetrant concentration. The orthoOAPS follows the metaOAPS but it is not able to swell as much, thus limiting the uptake. The paraOAPS is always the least dense structure and can thus sorb a lot of penetrants before it has to swell. However, its swelling ability is almost as good as the metaOAPS, which in turn further increases its sorption capacity. There is thus an added effect of the organic-inorganic connectivity in their swelling behaviour.

Radial distribution functions $\mathrm{g}(r)$ for the $\mathrm{C}$ in $\mathrm{CH}_{4}$ and the $\mathrm{O}$ in $\mathrm{CO}_{2}$ were used to characterize the actual interaction sites of the penetrants in the networks. A $g(r)$ between atom-types $i$ and $j$ gives the average probability of finding $n_{j}$ atoms of type $j$ around an atom of type $i$ within a spherical shell of volume $4 \pi r^{2} \delta r$ relative to that expected from a random distribution at the same number density for type $j, \rho_{\mathrm{j}}$ (Eq. (9)):

$g(r)=\frac{n_{j}}{4 \pi r^{2} \delta r \rho_{j}}$

The histograms are collected using bins of equal radial width and the $\mathrm{g}(r)$ are normalized accordingly taking into account the increase of the volume as $r$ increases. The closest interactions are the same as those in polyimides $[36,83,84,86,97]$, i.e. the penetrants easily approach the imide ketone oxygens and the fluorine groups on the 6FDA moiety. However, as seen in Fig. 6, there are also preferential sites in the vicinity of the siloxane cages and the OAPS phenyl rings. Representative $g(r)$ are compared at the same pressure (20 bar) in Fig. 11.

The $g(r)$ confirm that the penetrants occupy easily-accessible sites, which include those close to the protruding groups of the organic imide moieties and the pendant $\mathrm{NH}_{2}$ groups on the unreacted OAPS or POSS arms (Fig. 10c-e). There are also less-accessible sites in the immediate vicinity of the siloxane cages, i.e. close to the $\mathrm{O}$ in between the armbearing $\mathrm{Si}$ atoms, except for the orthoOAPS (Fig. 10a) where the reacted and unreacted $-\mathrm{NH}_{2}$ groups in the ortho position naturally occlude the space close to the cage. In general, the penetrants favour the organic phase in the POSS networks, which contains their lowest-energy sites (Fig. 6). On the other hand, the lowest-energy sites of the OAPS networks are closer to the cages and in the vicinity of their phenyl arm linkers (Fig. 11b). As expected, the behaviour of the penetrants in the paraOAPS and metaOAPS networks is quite similar, while the orthoOAPS differs because of its specific features. At higher pressures, the preferential interactions do not change as the general increase in the freevolume is affine with the amount of volume swelling (Figs. 9 and 10) $[36,84]$. There are no signs of permanent clusters forming, even if some penetrant molecules tend to find themselves close to others, especially at high pressures. Snapshots of $\mathrm{CH}_{4}$ sorbed in the polymetaOAPS-6FDA matrix and $\mathrm{CO}_{2}$ sorbed in the polyparaOAPS-PMDA matrix at different pressures are provided in Fig. 12. In addition, 3D animations of the polyparaOAPS-PMDA and polyorthoOAPS-PMDA simulation boxes loaded with 20 bar of $\mathrm{CO}_{2}$ can be found in the Supporting Information.

The ideal $\mathrm{CO}_{2} / \mathrm{CH}_{4}$ sorption selectivities can be obtained from the sixteen uptake curves (Fig. 8) as:

$\alpha_{\mathrm{CO}_{2} / \mathrm{CH}_{4}}^{S}(p)=\frac{C_{\text {matrix }-\mathrm{CO}_{2}}(p)}{C_{\text {matrix }-\mathrm{CH}_{4}}(p)}$

and they are given in Fig. 13.

Although it could have been expected that the lower densities and higher fractions of void-space in the polyOAPS-imides would lead to a significant decrease in the sorption selectivities, this is not the case. The selectivities are only slightly smaller than those in the denser polyPOSSimides and are still consistent with experimental $\mathrm{CO}_{2} / \mathrm{CH}_{4}$ sorption selectivities found in polyimides [110,111]. The sorption selectivity in these hyper-cross-linked networks thus does not seem to be affected by the set of inorganic and organic precursors studied here. There could be

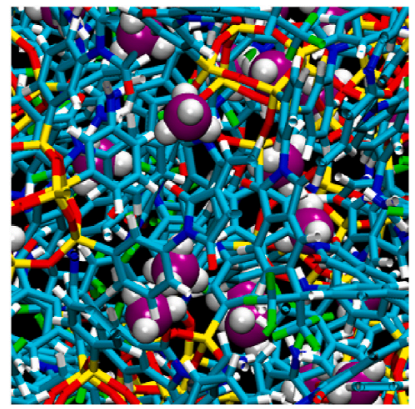

5 bar

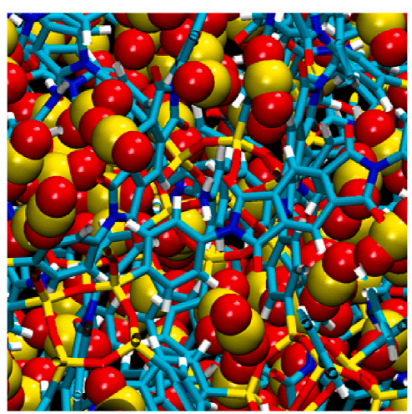

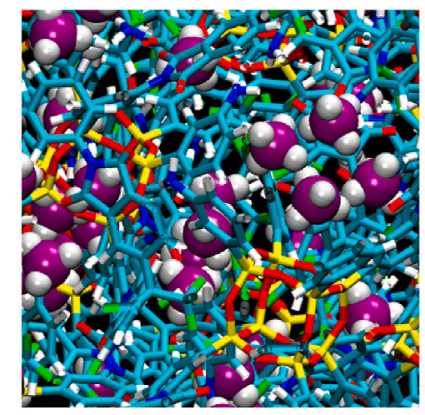

20 bar

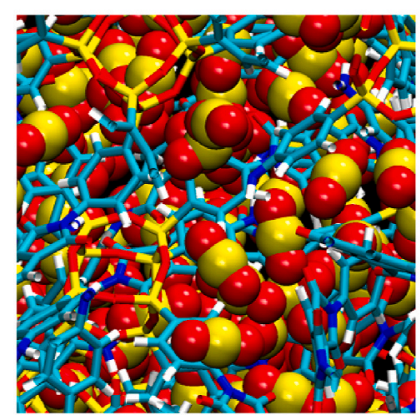

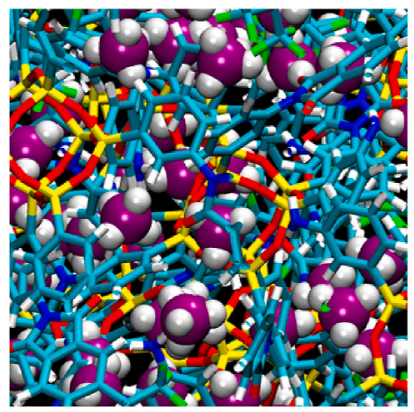

60 bar

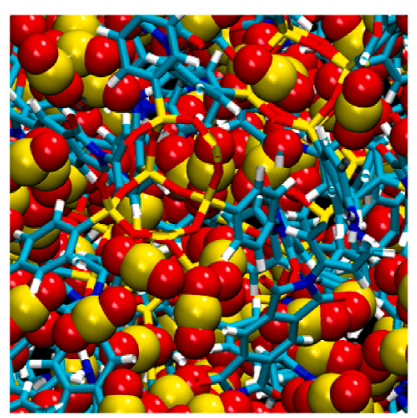

Fig. 12. $\sim(20 \AA)^{2}$ close-ups of the polymetaOAPS-6FDA network loaded with $\mathrm{CH}_{4}$ (top pictures) and the polyparaOAPS-6FDA network loaded with $\mathrm{CO}_{2}$ (bottom pictures). The colour code is the same as in Fig. 2. (For interpretation of the references to colour in this figure legend, the reader is referred to the Web version of this article.) 

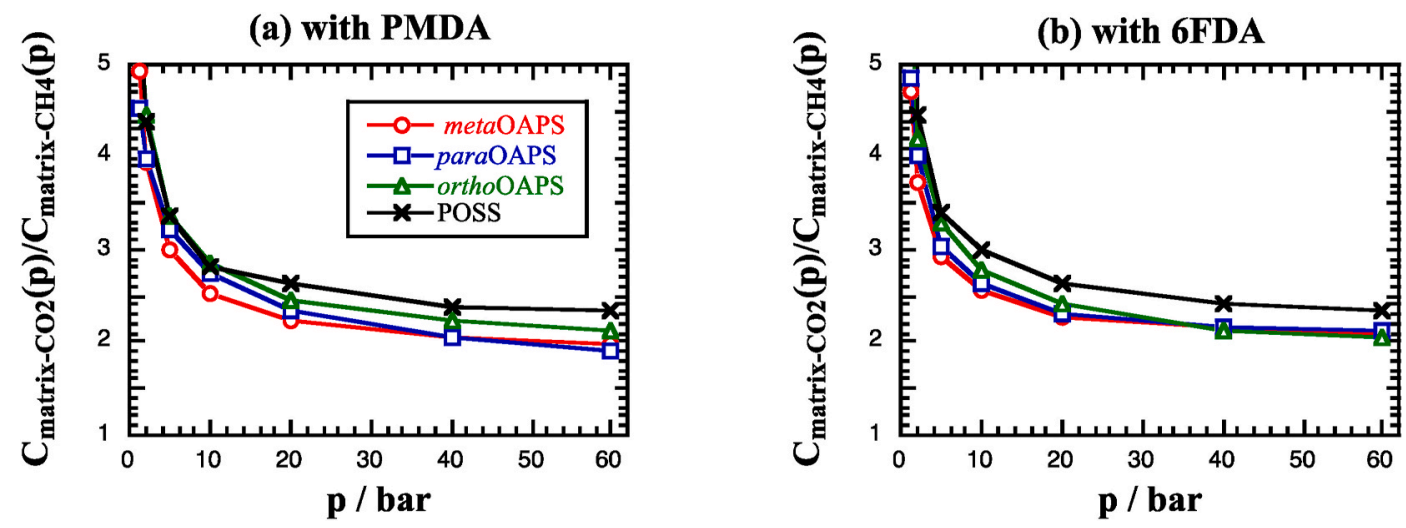

Fig. 13. Ideal $\mathrm{CO}_{2} / \mathrm{CH}_{4}$ sorption selectivities (Eq. (8)) at $35{ }^{\circ} \mathrm{C}$ for the eight networks under study.

larger differences in the diffusion selectivities and that will be the subject of future work.

\section{Mechanical resistances of the polyOAPS/POSS-imide networks}

In addition to the sorption-induced swelling, separation membranes need to be able to resist other mechanical stresses when subjected to harsh conditions. The network resistance to isotropic dilation and uniaxial tension has already been evaluated at high temperatures $\left(300{ }^{\circ} \mathrm{C}\right.$ and $400{ }^{\circ} \mathrm{C}$ ) during our initial screening stage [30], but we briefly present here the corresponding results at $35^{\circ} \mathrm{C}$, which were obtained from additional MD simulations. The isotropic volume dilation was tested by simultaneously reducing the $x x, y y$ and $z z$ on-diagonal components of the pressure tensor and the uniaxial extension by only changing the $y y$ component. In both cases, the rate of deformation was -1 bar/ps. In

\section{(a) isotropic dilation - PMDA}

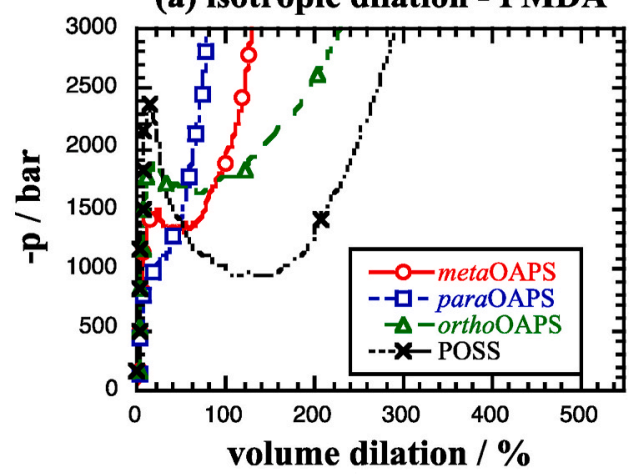

(c) tension - PMDA

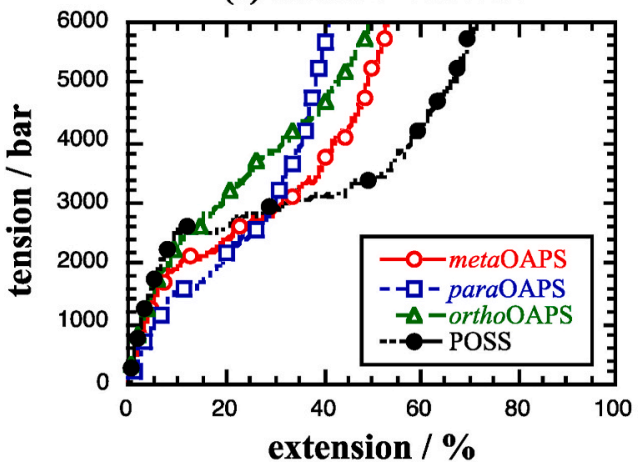

spite of the rates of deformation accessible to the MD timescale being very fast, such simulations are still able to provide a reasonable comparison of the intrinsic mechanical properties [26,27,112,113]. The resistances to isotropic dilation and uniaxial tension at $35{ }^{\circ} \mathrm{C}$ for the eight networks under study are given in Fig. 14.

Both tests show the same trends (Fig. 14). At the very start, the polyPOSS-imides resist better against the deformations as they are dense and have the smallest $<\% P A V>$ (Figs. 3 and 4). As such, they have the highest yield stresses in the dilation tests (Fig. 14a-b) and the highest elastic moduli in the tension tests (Fig. 14c-d). The model $E$ agree well with the experimental moduli reported for closely-related structures $[27,114,115]$. The initial deformation of the polyPOSS-imides is then followed by a ductile behaviour typical of cold-drawing and a subsequent strain hardening stage. On the other hand, the polyOAPS-imides strain harden at much lower values of the dilation/extension because of their phenyl linkers, thus strongly limiting the ductile stage. The

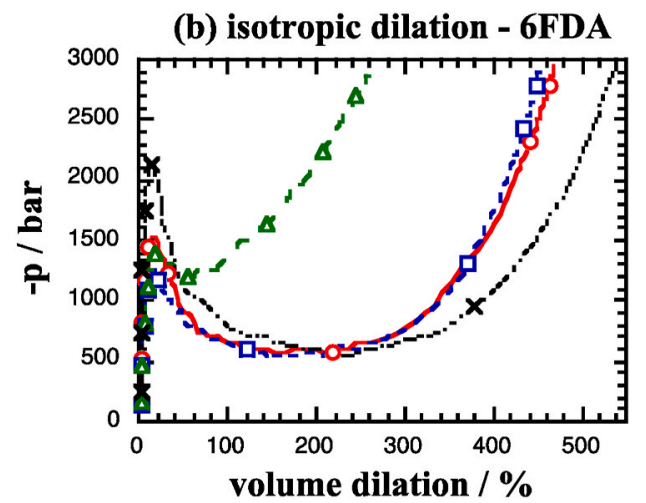

(d) Young's moduli - 6FDA

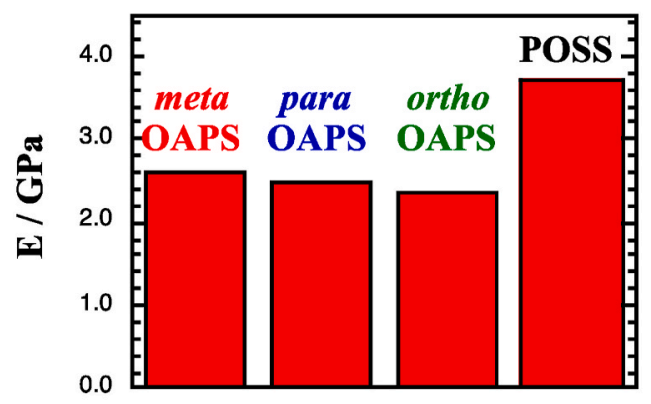

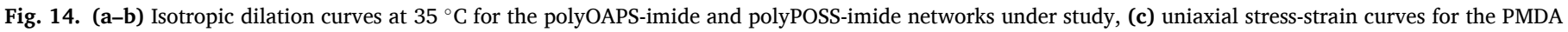
networks and (d) average Young's moduli $E$ for the 6FDA networks. The maximum standard error on E is $0.05 \mathrm{GPa}$. 
orthoOAPS tends to bring added resistance, as does the stiffer PMDA. For the flexible 6FDA, the organic linker is already extended when associated to orthoOAPS, unlike for the para and meta isomers where it has more scope for deformation (Table 2). However, in all cases, the polyOAPS-imides consistently outperform the polyPOSS-imides in terms of strain hardening, which confirms their better overall mechanical resistance.

\section{Conclusions}

In this work, the molecular characteristics and sorption capacities at $35{ }^{\circ} \mathrm{C}$ were compared for a series of hybrid hyper-cross-linked model networks based on cubic $\mathrm{Si}_{8} \mathrm{O}_{12}$ cages covalently bonded with imides, in which the inorganic linkers originate either from POSS (with aliphatic $-\left(\mathrm{CH}_{2}\right)_{3}-\mathrm{NH}_{2}$ arms) or from OAPS (with phenyl $-\mathrm{C}_{6} \mathrm{H}_{4}-\mathrm{NH}_{2}$ arms). PolyPOSS-imide membranes have already been synthesized using interfacial polycondensation on ceramic porous supports [18-21] and upgraded to tubular modules [25]. However, they cannot be used above $300{ }^{\circ} \mathrm{C}$ due to the decomposition of their aliphatic $-\left(\mathrm{CH}_{2}\right)_{3}-$ linkers $[21$, $26,27]$. Using the phenyl-arm OAPS as the inorganic precursor should increase their thermoresistance, but it has three possible isomers depending on the meta, para or ortho position of the $-\mathrm{NH}_{2}$ group on the phenyl ring.

To avoid a long programme of synthesis, a large number of molecular networks based on the three isomers of OAPS and on the PMDA, 6FDA and ODPA imides were recently modelled at different cross-link densities [30]. Their thermoresistance was screened at $300{ }^{\circ} \mathrm{C}$ and $400{ }^{\circ} \mathrm{C}$ and six promising networks, i.e. the three polyOAPS-PMDA and the three polyOAPS-6FDA with the maximum cross-link densities, were selected for further examination of their sorption properties at $35{ }^{\circ} \mathrm{C}$. These are compared here to those of the corresponding polyPOSS-imides generated under exactly the same conditions.

The network connectivities and morphologies are highly dependent on their precursors. All of them have an average number of $\sim 5$ links per cage, but these include intercage single-links and double-links as well as intracage links, thus reducing the actual cross-linking densities to an average of $\sim 3.8$. The metaOAPS and paraOAPS networks have rather similar $\mathrm{Si} \cdots \mathrm{Si}$ bridging distances and imide $\mathrm{N} \cdots$ centre $\cdots \mathrm{N}$ angles, unlike the ortho functionalization, which leads to connectivities closer to those of the POSS. Densities vary quite a lot, and differences are enhanced when the inorganic precursor is associated to the stiffer PMDA imide.

The TPI method, combined to the EVMS formalism for added efficiency, was used to obtain the infinite-dilution solubilities of $\mathrm{CH}_{4}$ and $\mathrm{CO}_{2}$ penetrant molecules as well as the underlying distributions of insertion energies. The free-volume morphology analyses showed that the phenyl-arm OAPS all result in more open structures than the POSS, although the orthoOAPS Boltzmann-weighted probability density distributions for the insertion energies of the penetrants are closer to POSS than to metaOAPS and paraOAPS. Both the latter have high solubilities because of very favourable sites in the spaces between the OAPS arms. On the other hand, orthoOAPS inherently prevents access to the cages and its low-energy sites tend to be close to the phenyl rings but further away from the cages. This is more similar to POSS, where the lowestenergy sites are spread in the organic phase.

The iterative GCMC-MD approach was used to predict both the $\mathrm{CH}_{4}$ and $\mathrm{CO}_{2}$ sorption isotherms at $35{ }^{\circ} \mathrm{C}$ over the $0-60$ bar pressure range in the eight networks under study. The sorption capacities varied in the order paraOAPS $>$ metaOAPS $>$ orthoOAPS $>$ POSS, in agreement with their larger amounts of accessible volume. Once again, the behaviour of orthoOAPS was closer to that of POSS, except that it had a significantly higher fraction of its volume available for penetrant insertion in the 6FDA networks. In all cases, systems with the OAPS precursor showed less swelling when compared at equivalent penetrant concentrations in the matrix. However, their $\mathrm{CO}_{2} / \mathrm{CH}_{4}$ ideal sorption selectivity remained essentially the same at equivalent gas pressures.

Further mechanical isotropic dilation and uniaxial tension tests confirmed the better strain hardening response of the polyOAPS-imides due to the phenyl arms. The polyOAPS-imides are thus interesting because of their better sorption capacities and their added resistance to mechanical deformation whilst still keeping a similar sorption selectivity to the polyPOSS-imides. Diffusion and transport properties at higher temperatures will be analysed in a future work.

\section{Declaration of competing interest}

The authors declare that they have no known competing financial interests or personal relationships that could have appeared to influence the work reported in this paper.

\section{Acknowledgments}

This work is part of the MOLHYB project financed by the French ANR (Agence Nationale de la Recherche) within the framework of the AAPG (Appel à Projets Générique) 2018. It had access to the HPC resources of CCRT/CINES/IDRIS under the allocations A007-and A009-095053 made by GENCI, France. The MUST computing centre at the University Savoie Mont Blanc, France, is also acknowledged for the provision of computer time. Nicolas Charvin is thanked for his help with the installation and maintenance of the laboratory local servers.

\section{Appendix A. Supplementary data}

Supplementary data to this article can be found online at https://doi. org/10.1016/j.memsci.2021.119531.

\section{CRediT author statement}

S. Neyertz: Conceptualization, Funding Acquisition, Resources, Methodology, Investigation, Formal Analysis, Writing - original draft, D. Brown: Software, Methodology, Investigation, Formal Analysis, Writing - review\&editing, S. Salimi: Writing - review\&editing. F. Radmanesh: Writing - review\&editing. N.E. Benes: Conceptualization, Funding Acquisition, Writing - review\&editing.

\section{References}

[1] D.S. Sholl, R.P. Lively, Seven chemical separations to change the world, Nature 532 (2016) 435-437, https://doi.org/10.1038/532435a.

[2] Y. Yampolskii, I. Pinnau, B.D. Freeman, Materials Science of Membranes for Gas and Vapor Separation, John Wiley \& Sons Ltd., Chichester, UK, 2006.

[3] M. Galizia, W.S. Chi, Z.P. Smith, T.C. Merkel, R.W. Baker, B.D. Freeman, 50th anniversary perspective: polymers and mixed matrix membranes for gas and vapor separation: a review and prospective opportunities, Macromolecules 50 (2017) 7809-7843, https://doi.org/10.1021/acs.macromol.7b01718.

[4] J. Hennessy, Membranes from academia to industry, Nat. Mater. 16 (2017) 280-282, https://doi.org/10.1038/nmat4861.

[5] L.M. Robeson, The upper bound revisited, J. Membr. Sci. 320 (2008) 390-400, https://doi.org/10.1016/j.memsci.2008.04.030.

[6] B.W. Rowe, L.M. Robeson, B.D. Freeman, D.R. Paul, Influence of temperature on the upper-bound: theoretical considerations and comparison with experimental results, J. Membr. Sci. 360 (2010) 58-69, https://doi.org/10.1016/j. memsci.2010.04.047.

[7] H.J. Kuenen, H.J. Mengers, A. Nijmeijer, A.G.J. Van der Ham, A.A. Kiss, Technoeconomic evaluation of the direct conversion of $\mathrm{CO}_{2}$ to dimethyl carbonate using catalytic membrane reactors, Comput. Chem. Eng. 86 (2016) 136-147, https:// doi.org/10.1016/j.compchemeng.2015.12.025.

[8] B.S.R. Reddy, Advances in Nanocomposites - Synthesis, Characterization and Industrial Applications, InTech, Rijeka, Croatia, 2011.

[9] D.B. Cordes, P.D. Lickiss, F. Rataboul, Recent developments in the chemistry of cubic polyhedral oligosilsesquioxanes, Chem. Rev. 110 (2010) 2081-2173, https://doi.org/10.1021/cr900201r.

[10] R.M. Laine, M.F. Roll, Polyhedral phenylsilsesquioxanes, Macromolecules 44 (2011) 1073-1109, https://doi.org/10.1021/ma102360t.

[11] Y. Du, H. Liu, Cage-like silsesquioxanes-based hybrid materials, Dalton Trans. 49 (2020) 5396-5405, https://doi.org/10.1039/D0DT00587H.

[12] P. Iyer, G. Iyer, M. Coleman, Gas transport properties of polyimide-POSS nanocomposites, J. Membr. Sci. 358 (2010) 26-32, https://doi.org/10.1016/j. memsci.2010.04.023.

[13] D.M. Pinson, G.R. Yandek, T.S. Haddad, E.M. Horstman, J.M. Mabry, Thermosetting poly(imide silsesquioxane)s featuring reduced moisture affinity 
and improved processability, Macromolecules 46 (2013) 7363-7377, https://doi. org $/ 10.1021 / \mathrm{ma} 401347$.

[14] K.N. Raftopoulos, K. Pielichowski, Segmental dynamics in hybrid polymer/POSS nanomaterials, Prog. Polym. Sci. 52 (2016) 136-187, https://doi.org/10.1016/j progpolymsci.2015.01.003.

[15] M.G. Mohamed, S.-W. Kuo, Functional polyimide/polyhedral oligomeric silsesquioxane nanocomposites, Polymers 11 (2019) 26, https://doi.org/ 10.3390/polym 11010026 .

[16] Z. Lan, X. Chen, X. Zhang, C. Zhu, Y. Yu, J. Wei, Transparent, high glass-transition temperature, shape memory hybrid polyimides based on polyhedral oligomeric silsesquioxane, Polymers 11 (2019) 1058, https://doi.org/10.3390/ polym 11061058.

[17] X. Chen, L.F. Dumée, Polyhedral oligomeric silsesquioxane (POSS) nanocomposite separation membranes - A review, Adv. Eng. Mater. 21 (2019), 1800667, https://doi.org/10.1002/adem.201800667.

[18] M.J.T. Raaijmakers, M. Wessling, A. Nijmeijer, N.E. Benes, Hybrid polyhedral oligomeric silsesquioxanes-imides with tailored intercage spacing for sieving of hot gases, Chem. Mater. 26 (2014) 3660-3664, https://doi.org/10.1021/ cm500691e.

[19] M.J.T. Raaijmakers, M.A. Hempenius, P.M. Schön, G.J. Vancso, A. Nijmeijer, M. Wessling, N.E. Benes, Sieving of hot gases by hyper-cross-linked nanoscalehybrid membranes, J. Am. Chem. Soc. 136 (2014) 330-335, https://doi.org/ 10.1021/ja410047u.

[20] M.J.T. Raaijmakers, W. Ogieglo, M. Wiese, M. Wessling, A. Nijmeijer, N.E. Benes, Sorption behavior of compressed $\mathrm{CO}_{2}$ and $\mathrm{CH}_{4}$ on ultrathin hybrid poly(POSSimide) layers, ACS Appl. Mater. Interfaces 7 (2015) 26977-26988, https://doi. org/10.1021/acsami.5b08286.

[21] M.J.T. Raaijmakers, E.J. Kappert, A. Nijmeijer, N.E. Benes, Thermal imidization kinetics of ultrathin films of hybrid poly(POSS-imide)s, Macromolecules 48 (2015) 3031-3039, https://doi.org/10.1021/acs.macromol.5b00473.

[22] M.K. Ghosh, K.L. Mittal, Polyimides: Fundamentals and Applications, Marcel Dekker, Inc., New York, 1996.

[23] Y. Yampolskii, Polymeric gas separation membranes, Macromolecules 45 (2012) 3298-3311, https://doi.org/10.1021/ma300213b.

[24] E. Maaskant, P. De Wit, N.E. Benes, Direct interfacial polymerization onto thin ceramic hollow fibers, J. Membr. Sci. 550 (2018) 296-301, https://doi.org/ 10.1016/j.memsci.2018.01.009.

[25] L. Ansaloni, E. Louradour, F. Radmanesh, H. van Veen, M. Pilz, C. Simon, N. E. Benes, T.A. Peters, Upscaling polyPOSS-imide membranes for high temperature $\mathrm{H}_{2}$ upgrading, J. Membr. Sci. 620 (2021), 118875, https://doi.org/10.1016/j. memsci.2020.118875.

[26] S. Neyertz, D. Brown, M.J.T. Raaijmakers, N.E. Benes, A molecular characterization of hyper-cross-linked hybrid polyPOSS-imide networks, Comput. Mater. Sci. 117 (2016) 338-353, https://doi.org/10.1016/j. commatsci.2016.02.015.

[27] S. Neyertz, D. Brown, M.J.T. Raaijmakers, N.E. Benes, The influence of the dianhydride precursor in hyper-cross-linked hybrid polyPOSS-imide networks, Phys. Chem. Chem. Phys. 18 (2016) 28688-28703, https://doi.org/10.1039/ C6CP06184B.

[28] D. Brown, S. Neyertz, M.J.T. Raaijmakers, N.E. Benes, Sorption and permeation of gases in hyper-cross-linked hybrid poly(POSS-imide) networks: an in silico study, J. Membr. Sci. 577 (2019) 113-119, https://doi.org/10.1016/j. memsci.2019.01.039.

[29] R. Tamaki, Y. Tanaka, M.Z. Asuncion, J. Choi, R.M. Laine, Octa(aminophenyl) silsesquioxane as a nanoconstruction site, J. Am. Chem. Soc. 123 (2001) 12416-12417, https://doi.org/10.1021/ja011781m.

[30] S. Neyertz, S. Salimi, F. Radmanesh, N.E. Benes, D. Brown, High-temperature molecular screening of hybrid polyOAPS-imide networks based on octa (aminophenyl)silsesquioxane for increased thermomechanical resistance, Phys. Chem. Chem. Phys. 23 (2021) 11438-11454, https://doi.org/10.1039/ D1CP01052B.

[31] S.G. Kim, J. Choi, R. Tamaki, R.M. Laine, Synthesis of amino-containing oligophenylsilsesquioxanes, Polymer 46 (2005) 4514-4524, https://doi.org/ 10.1016/j.polymer.2005.02.036.

[32] J.K. Koech, Q. Shao, F.N. Mutua, Y. Wang, Application of hydrazine hydrate in the synthesis of octa(aminophenyl)silsesquioxane (OAPS) Poss, Adv. Chem. Eng Sci. 3 (2013) 93-97, https://doi.org/10.4236/aces.2013.31011.

[33] A. Lee, T.S. Haddad, J.J. Schwab, Y.Z. An, Molecular structure analysis of aminophenyl silsesquioxanes, available at, https://www.researchgate.net/pu blication/277880144, 2006

[34] D.M. Anstine, A.G. Demidov, N.F. Mendez, W.J. Morgan, C.M. Colina, Screening PIM-1 performance as a membrane for binary mixture separation of gaseous organic compounds, J. Membr. Sci. 599 (2020), 117798, https://doi.org/ 10.1016/j.memsci.2019.117798.

[35] M.J.T. Raaijmakers, Hyper-cross-linked, Hybrid Membranes via Interfacial Polymerization, Doctoral Thesis, University of Twente, The Netherlands, 2015.

[36] S. Neyertz, D. Brown, Single- and mixed-gas sorption in large-scale molecular models of glassy bulk polymers. Competitive sorption of a binary $\mathrm{CH}_{4} / \mathrm{N}_{2}$ and a ternary $\mathrm{CH}_{4} / \mathrm{N}_{2} / \mathrm{CO}_{2}$ mixture in a polyimide membrane, J. Membr. Sci. 614 (2020), 118478, https://doi.org/10.1016/j.memsci.2020.118478.

[37] S. Nagendiran, A. Chandramohan, K. Dinakaran, M. Alagar, Octahedral oligomeric silsesquioxane (OAPS and OG) - polyimide hybrid nanocomposite films: thermo-mechanical, dielectric and morphology properties, J. Macromol. Sci., Pure Appl. Chem. 56 (2019) 1082-1096, https://doi.org/10.1080/ 10601325.2019.1653197.
[38] J. Yao, S. Ma, J. Zhang, Y. Wang, C. Wang, H. Zhou, C. Chen, G. Liu, Multiple shape memory effects of polyimide nanocomposites based on octa(aminophenyl) silsequioxanes, Express Polym. Lett. 15 (2021) 433-444, https://doi.org/ 10.3144/expresspolymlett.2021.37.

[39] J.M. Stubbs, B. Chen, J.J. Potoff, J.I. Siepmann, Monte Carlo calculations for the phase equilibria of alkanes, alcohols, water and their mixtures, Fluid Phase Equil. 183-184 (2001) 301-309, https://doi.org/10.1016/S0378-3812(01)00442-3.

[40] C. Rizzuto, A. Caravella, A. Brunetti, C.H. Park, Y.M. Lee, E. Drioli, G. Barbieri, E. Tocci, Sorption and diffusion of $\mathrm{CO}_{2} / \mathrm{N}_{2}$ in gas mixture in thermally-rearranged polymeric membranes: a molecular investigation, J. Membr. Sci. 528 (2017) 135-146, https://doi.org/10.1016/j.memsci.2017.01.025.

[41] A. Brunetti, E. Tocci, M. Cersocimo, J.S. Kim, W.H. Lee, J.G. Seong, Y.M. Lee, E. Drioli, G. Barbieri, Mutual influence of mixed-gas permeation in thermally rearranged poly(benzoxazole-co-imide) polymer membranes, J. Membr. Sci. 580 (2019) 202-213, https://doi.org/10.1016/j.memsci.2019.01.058.

[42] D.M. Anstine, C.M. Colina, Sorption-induced polymer rearrangement: approaches from molecular modeling, Polym. Int 70 (2020) 984-989. https://doi.org/10.100 2/pi.6124.

[43] R. Chatterjee, A.G. Kumar, R. Nikiforov, V. Ryzhikh, N. Belov, V. Padmanabhan, Y. Yampolskii, S. Banerjee, Novel semi-fluorinated poly(ether imide)s with benzyl ether side groups: synthesis, physicochemical characterization, gas transport properties and simulation, Eur. Polym. J. 135 (2020), 109879, https://doi.org/ 10.1016/j.eurpolymj.2020.109879.

[44] F. Amirkhani, H.R. Harami, M. Asghari, $\mathrm{CO}_{2} / \mathrm{CH}_{4}$ mixed gas separation using poly (ether-b-amide)-ZnO nanocomposite membranes: experimental and molecular dynamics study, Polym. Test. 86 (2020), 106464, https://doi.org/10.1016/j. polymertesting.2020.106464.

[45] G. Kupgan, A.G. Demidov, C.M. Colina, Plasticization behavior in polymers of intrinsic microporosity (PIM-1): a simulation study from combined Monte Carlo and molecular dynamics, J. Membr. Sci. 565 (2018) 95-103, https://doi.org/ 10.1016/j.memsci.2018.08.004.

[46] S. Velioglu, M.G. Ahunbay, S.B. Tantekin-Ersolmaz, Investigation of $\mathrm{CO}_{2}$-induced plasticization in fluorinated polyimide membranes via molecular simulation, J. Membr. Sci. 417-418 (2012) 217-227, https://doi.org/10.1016/j memsci.2012.06.043.

[47] S. Velioglu, M.G. Ahunbay, S.B. Tantekin-Ersolmaz, An atomistic insight on $\mathrm{CO}_{2}$ plasticization resistance of thermally rearranged 6FDA-bisAPAF, J. Membr. Sci. 556 (2018) 23-33, https://doi.org/10.1016/j.memsci.2018.03.047.

[48] M. Balçik, M.G. Ahunbay, Prediction of $\mathrm{CO}_{2}$-induced plasticization pressure in polyimides via atomistic simulations, J. Membr. Sci. 547 (2018) 146-155, https://doi.org/10.1016/j.memsci.2017.10.038.

[49] P.K. Roy, K. Kumar, F.M. Thakkar, A.D. Pathak, K.G. Ayappa, P.K. Maiti, Investigations on 6FDA/BPDA-DAM polymer melt properties and $\mathrm{CO}_{2}$ adsorption using molecular dynamics simulations, J. Membr. Sci. 613 (2020), 118377, https://doi.org/10.1016/j.memsci.2020.118377.

[50] M. Balçik, S.B. Tantekin-Ersolmaz, M.G. Ahunbay, Interfacial analysis of mixedmatrix membranes under exposure to high-pressure $\mathrm{CO}_{2}$, J. Membr. Sci. 607 (2020), 118147, https://doi.org/10.1016/j.memsci.2020.118147.

[51] D. Tang, G. Kupgan, C.M. Colina, Rapid prediction of adsorption isotherms of a diverse range of molecules in hyper-cross-linked polymers, J. Phys. Chem. C 123 (2019) 17884-17893, https://doi.org/10.1021/acs.jpcc.9b04413.

[52] D. Brown, The Gmq User Manual Version 6, 2021 available at, http://www. lmops.univ-savoie.fr/brown/gmq.html.

[53] D. Yin, A.D. MacKerell Jr., Combined ab initio/empirical approach for optimization of Lennard-Jones parameters, J. Comput. Chem. 19 (1998) 334-348, https://doi.org/10.1002/(SICI)1096-987X(199802)19:3\%3C334::AID JCC7\%3E3.0.CO;2-U.

[54] Z. Zhang, Z. Duan, An optimized molecular potential for carbon dioxide, J. Chem. Phys. 122 (2005), 214507, https://doi.org/10.1063/1.1924700.

[55] G. Ciccotti, M. Ferrario, J.P. Ryckaert, Molecular dynamics of rigid systems in cartesian coordinates. A general formulation, Mol. Phys. 47 (1982) 1253-1264, https://doi.org/10.1080/00268978200100942.

[56] K.D. Hammonds, J.-P. Ryckaert, On the convergence of the SHAKE algorithm, Comput. Phys. Commun. 62 (1991) 336-351, https://doi.org/10.1016/00104655(91)90105-T.

[57] D.J. Tildesley, P. Madden, An effective pair potential for liquid carbon disulphide, Mol. Phys. 42 (1981) 1137-1156, https://doi.org/10.1080/ 00268978100100861.

[58] H.J.C. Berendsen, J.P.M. Postma, W.F. Van Gunsteren, A. DiNola, J.R. Haak, Molecular dynamics with coupling to an external bath, J. Chem. Phys. 81 (1984) 3684-3690, https://doi.org/10.1063/1.448118.

[59] D. Brown, J.H.R. Clarke, A loose-coupling constant pressure molecular dynamics algorithm for use in the modelling of polymer materials, Comput. Phys. Commun. 62 (1991) 360-369, https://doi.org/10.1016/0010-4655(91)90107-V.

[60] W. Humphrey, A. Dalke, K. Schulten, VMD: visual molecular dynamics, J. Mol. Graph. 14 (1996) 33-38, https://doi.org/10.1016/0263-7855(96)00018-5.

[61] C. Wu, W. Xu, Atomistic molecular modelling of crosslinked epoxy resin, Polymer 47 (2006) 6004-6009, https://doi.org/10.1016/j.polymer.2006.06.025.

[62] V. Varshney, S.S. Patnaik, A.K. Roy, B.L. Farmer, A molecular dynamics study of epoxy-based networks: cross-linking procedure and prediction of molecular and material properties, Macromolecules 41 (2008) 6837-6842, https://doi.org/ 10.1021/ma801153e.

[63] N. Lacevic, R.H. Gee, A. Saab, R. Maxwell, Computational exploration of polymer nanocomposite mechanical property modification via cross-linking topology, J. Chem. Phys. 129 (2008), 124903, https://doi.org/10.1063/1.2980044. 
[64] P.-H. Lin, R. Khare, Molecular simulation of cross-linked epoxy and epoxy-POSS nanocomposite, Macromolecules 42 (2009) 4319-4327, https://doi.org/ 10.1021/ma9004007.

[65] P.-H. Lin, R. Khare, Glass transition and structural properties of glycidyloxypropyl-heptaphenyl polyhedral oligomeric silsesquioxane-epoxy nanocomposites. A molecular simulation study, J. Therm. Anal. Calorim. 102 (2010) 461-467, https://doi.org/10.1007/s10973-010-0982-7.

[66] N.B. Shenogina, M. Tsige, S.S. Patnaik, S.M. Mukhopadhyay, Molecular modeling approach to prediction of thermo-mechanical behavior of thermoset polymer networks, Macromolecules 45 (2012) 5307-5315, https://doi.org/10.1021/ ma3007587.

[67] L.J. Abbott, C.M. Colina, Atomistic structure generation and gas adsorption simulations of microporous polymer networks, Macromolecules 44 (2011) 4511-4519, https://doi.org/10.1021/ma200303p.

[68] A. Bandyopadhyay, P.K. Valavala, T.C. Clancy, K.E. Wise, G.M. Odegard, Molecular modeling of crosslinked epoxy polymers: the effect of crosslink density on thermomechanical properties, Polymer 52 (2011) 2445-2452, https://doi. org/10.1016/j.polymer.2011.03.052.

[69] J.H. Moon, A.R. Katha, S. Pandian, S.M. Kolake, S. Han, Polyamide-POSS hybrid membranes for seawater desalination: effect of POSS inclusion on membrane properties, J. Membr. Sci. 461 (2014) 89-95, https://doi.org/10.1016/j. memsci.2014.03.004.

[70] C. Jang, T.W. Sirk, J.W. Andzelm, C.F. Abrams, Comparison of crosslinking algorithms in molecular dynamics simulation of thermosetting polymers, Macromol. Theory Simul. 24 (2015) 260-270, https://doi.org/10.1002/ mats.201400094.

[71] S. Pandiyan, J. Krajniak, G. Samaey, D. Roose, E. Nies, A molecular dynamics study of water transport inside an epoxy polymer matrix, Comput. Mater. Sci. 106 (2015) 29-37, https://doi.org/10.1016/j.commatsci.2015.04.032.

[72] Y. Sun, Y. Guo, H. Yang, A molecular dynamics study of crosslinked epoxy networks: construction of atomistic models, Mol. Simulat. 46 (2020) 121-127, https://doi.org/10.1080/08927022.2019.1679364.

[73] S.V. Lyulin, S.V. Larin, A.A. Gurtovenko, V.M. Nazarychev, S.G. Falkovich, V. E. Yudin, V.M. Svetlichnyi, I.V. Gofman, A.V. Lyulin, Thermal properties of bulk polyimides: insights from computer modeling versus experiment, Soft Matter 10 (2014) 1224-1232, https://doi.org/10.1039/c3sm52521j.

[74] F. Müller-Plathe, Permeation of polymers. A computational approach, Acta Polym. 45 (1994) 259-293, https://doi.org/10.1002/actp.1994.010450401.

[75] B. Widom, Some topics in theory of fluids, J. Chem. Phys. 39 (1963) 2808-2812, https://doi.org/10.1063/1.1734110.

[76] D. Frenkel, B. Smit, Understanding Molecular Simulation: from Algorithms to Applications, second ed., Academic Press, San Diego, CA and London, 2002.

[77] G.L. Deitrick, L.E. Scriven, H.T. Davis, Efficient molecular simulation of chemical potentials, J. Chem. Phys. 90 (1989) 2370-2385, https://doi.org/10.1063/ 1.455979 .

[78] Y. Tamai, H. Tanaka, K. Nakanishi, Molecular simulation of permeation of small penetrants through membranes. 2. Solubilities, Macromolecules 28 (1995) 2544-2554, https://doi.org/10.1021/ma00111a058.

[79] G. Dömötör, R. Hentschke, Atomistically modeling the chemical potential of small molecules in dense systems, J. Phys. Chem. B 108 (2004) 2413-2417, https://doi. org/10.1021/jp0367427.

[80] S. Pandiyan, D. Brown, S. Neyertz, N.F.A. Van der Vegt, Carbon dioxide solubility in three fluorinated polyimides studied by molecular dynamics simulations, Macromolecules 43 (2010) 2605-2621, https://doi.org/10.1021/ma902507d.

[81] I. Tanis, D. Brown, S. Neyertz, R. Heck, R. Mercier, M. Vaidya, J.-P. Ballaguet, A comparison of pure and mixed-gas permeation of nitrogen and methane in 6FDA-based polyimides as studied by molecular dynamics simulations, Comput. Mater. Sci. 141 (2018) 243-253, https://doi.org/10.1016/j. commatsci.2017.09.028.

[82] S. Neyertz, D. Brown, An optimized fully-atomistic procedure to generate glassy polymer films for molecular dynamics simulations, Comput. Mater. Sci. 174 (2020), 109499, https://doi.org/10.1016/j.commatsci.2019.109499.

[83] S. Neyertz, D. Brown, Molecular dynamics study of carbon dioxide sorption and plasticization at the interface of a glassy polymer membrane, Macromolecules 46 (2013) 2433-2449, https://doi.org/10.1021/ma302073u.

[84] S. Neyertz, D. Brown, The effect of structural isomerism on carbon dioxide sorption and plasticization at the interface of a glassy polymer membrane, J. Membr. Sci. 460 (2014) 213-228, https://doi.org/10.1016/j. memsci.2014.03.002

[85] S. Neyertz, D. Brown, Nanosecond-time-scale reversibility of dilation induced by carbon dioxide sorption in glassy polymer membranes, J. Membr. Sci. 520 (2016) 385-399, https://doi.org/10.1016/j.memsci.2016.08.003.

[86] S. Neyertz, D. Brown, Air sorption and separation by polymer films at the molecular level, Macromolecules 51 (2018) 7077-7092, https://doi.org/ 10.1021/acs.macromol.8b01423.

[87] M.P. Allen, D.J. Tildesley, Computer Simulation of Liquids, Clarendon Press, Oxford, UK, 1987.

[88] N.F.A. Van der Vegt, W.J. Briels, M. Wessling, H. Strathmann, The sorption induced glass transition in amorphous glassy polymers, J. Chem. Phys. 110 (1999) 11061-11069, https://doi.org/10.1063/1.479042.

[89] A. Ben-Naim, Y. Marcus, Solvation thermodynamics of nonionic solutes, J. Chem. Phys. 81 (1984) 2016-2027, https://doi.org/10.1063/1.447824.

[90] S. Neyertz, Gas Transport in Dense Polymeric Membranes, Molecular Dynamics Simulations, in: E.M.V. Hoek, V.V. Tarabara (Eds.), Encyclopedia of Membrane Science and Technology, John Wiley \& Sons, Hoboken, NJ, 2013.
[91] M. Mazo, N. Balabaev, A. Alentiev, I. Strelnikov, Y. Yampolskii, Structure and properties of high and low free volume polymers studied by molecular dynamics simulation, Computation 7 (2019) 27, https://doi.org/10.3390/ computation7020027.

[92] P.V.K. Pant, R.H. Boyd, Molecular dynamics simulation of diffusion of small penetrants in polymers, Macromolecules 26 (1993) 679-686, https://doi.org/ 10.1021/ma00056a019.

[93] A. Bondi, Van der Waals volumes and radii, J. Phys. Chem. 68 (1964) 441-451, https://doi.org/10.1021/j100785a001.

[94] L.M. Robeson, B.D. Freeman, D.R. Paul, B.W. Rowe, An empirical correlation of gas permeability and permselectivity in polymers and its theoretical basis, J. Membr. Sci. 341 (2009) 178-185, https://doi.org/10.1016/j. memsci.2009.06.005.

[95] R. Kirchheim, Sorption and partial molar volume of small molecules in glassy polymers, Macromolecules 25 (1992) 6952-6960, https://doi.org/10.1021/ ma00051a036.

[96] P. Gotthardt, A. Grüger, H.G. Brion, R. Plaetschke, R. Kirchheim, Volume change of glassy polymers by sorption of small molecules and its relation to the intermolecular space, Macromolecules 30 (1997) 8058-8065, https://doi.org/ $10.1021 / \mathrm{ma} 9708252$.

[97] S. Neyertz, D. Brown, S. Pandiyan, N.F.A. Van der Vegt, Carbon dioxide diffusion and plasticization in fluorinated polyimides, Macromolecules 43 (2010) 7813-7827, https://doi.org/10.1021/ma1010205.

[98] E. Ricci, M. Minelli, M.G. De Angelis, A multiscale approach to predict the mixed gas separation performance of glassy polymeric membranes for $\mathrm{CO}_{2}$ capture: the case of $\mathrm{CO}_{2} / \mathrm{CH}_{4}$ mixture in Matrimid $($, J. Membr. Sci. 539 (2017) 88-100, https://doi.org/10.1016/j.memsci.2017.05.068.

[99] E. Ricci, M.G. De Angelis, Modelling mixed-gas sorption in glassy polymers for $\mathrm{CO}_{2}$ removal: a sensitivity analysis of the Dual Mode Sorption model, Membranes 9 (2019) 8, https://doi.org/10.3390/membranes9010008.

[100] M.D. Donohue, G.L. Aranovich, Classification of Gibbs adsorption isotherms, Adv. Colloid Interface Sci. 76-77 (1998) 137-152, https://doi.org/10.1016/S00018686(98)00044-X.

[101] D.R. Paul, Gas sorption and transport in glassy polymers, Ber. Bunsen Ges. 83 (1979) 294-302, https://doi.org/10.1002/bbpc.19790830403.

[102] L.M. Robeson, Z.P. Smith, B.D. Freeman, D.R. Paul, Contributions of diffusion and solubility selectivity to the upper bound analysis for glassy gas separation membranes, J. Membr. Sci. 453 (2014) 71-83, https://doi.org/10.1016/j. memsci.2013.10.066.

[103] M. Böhning, J. Springer, Sorptive dilation and relaxational processes in glassy polymer/gas systems - I. Poly(sulfone) and poly(ether sulfone), Polymer 39 (1998) 5183-5195, https://doi.org/10.1016/S0032-3861(97)10114-8.

[104] M. Wessling, I. Huisman, T. Van der Boomgaard, C.A. Smolders, Dilation kinetics of glassy, aromatic polyimides induced by carbon dioxide sorption, J. Polym. Sci., Part B: Polym. Phys. 33 (1995) 1371-1384, https://doi.org/10.1002/ polb.1995.090330907.

[105] J.D. Wind, S.M. Sirard, D.R. Paul, P.F. Green, K.P. Johnston, W.J. Koros, Carbon dioxide-induced plasticization of polyimide membranes: pseudo-equilibrium relationships of diffusion, sorption, and swelling, Macromolecules 36 (2003) 6433-6441, https://doi.org/10.1021/ma0343582.

[106] W. Ogieglo, H. Wormeester, K.J. Eichhorn, M. Wessling, N.E. Benes, In situ ellipsometry studies on swelling of thin polymer films: a review, Prog. Polym. Sci. 42 (2015) 42-78, https://doi.org/10.1016/j.progpolymsci.2014.09.004.

[107] K. Simons, K. Nijmeijer, J. Guilera Sala, H. Van der Werf, N.E. Benes, T. J. Dingemans, $\mathrm{M}$. Wessling, $\mathrm{CO}_{2}$ sorption and transport behavior of ODPA-based polyetherimide polymer films, Polymer 51 (2010) 3907-3917, https://doi.org/ 10.1016/j.polymer.2010.06.031.

[108] A. Bos, I.G.M. Pünt, M. Wessling, H. Strathmann, $\mathrm{CO}_{2}$-induced plasticization phenomena in glassy polymers, J. Membr. Sci. 155 (1999) 67-78, https://doi. org/10.1016/S0376-7388(98)00299-3.

[109] K.L. Gleason, Z.P. Smith, Q. Liu, D.R. Paul, B.D. Freeman, Pure- and mixed-gas permeation of $\mathrm{CO}_{2}$ and $\mathrm{CH}_{4}$ in thermally rearranged polymers based on 3,3'dihydroxy-4,4'-diamino-biphenyl (HAB) and 2,2'-bis-(3,4-dicarboxyphenyl) hexafluoropropane dianhydride (6FDA), J. Membr. Sci. 475 (2015) 204-214, https://doi.org/10.1016/j.memsci.2014.10.014.

[110] M.R. Coleman, Isomers of Fluorine-Containing Polyimides for Gas Separation Membranes, Doctoral Thesis, University of Texas, Austin, TX, 1992.

[111] R. Wang, C. Cao, T.-S. Chung, A critical review on diffusivity and the characterization of diffusivity of 6FDA-6FpDA polyimide membranes for gas separation, J. Membr. Sci. 198 (2002) 259-271, https://doi.org/10.1016/S03767388(01)00665-2.

[112] A.V. Lyulin, M.A.J. Michels, Time scales and mechanisms of relaxation in the energy landscape of polymer glass under deformation: direct atomistic modeling, Phys. Rev. Lett. 99 (2007), 085504, https://doi.org/10.1103/ PhysRevLett.99.085504.

[113] C. Li, A. Strachan, Molecular dynamics predictions of thermal and mechanical properties of thermoset polymer EPON862/DETDA, Polymer 52 (2011) 2920-2928, https://doi.org/10.1016/j.polymer.2011.04.041.

[114] G. Ragosta, M. Abbate, P. Musto, G. Scarinzi, Effect of the chemical structure of aromatic polyimides on their thermal aging, relaxation behavior and mechanical properties, J. Mater. Sci. 47 (2012) 2637-2647, https://doi.org/10.1007/s10853011-6089-0.

[115] J. Choi, R. Tamaki, S.G. Kim, R.M. Laine, Organic/inorganic imide nanocomposites from aminophenylsilsesquioxanes, Chem. Mater. 15 (2003) 3365-3375, https://doi.org/10.1021/cm030286h. 